Boise State University

ScholarWorks

Mathematics Faculty Publications and

Presentations

Department of Mathematics

$10-9-2009$

\title{
Stability and Error Estimates for Vector Field Interpolation and Decomposition on the Sphere with RBFs
}

Edward J. Fuselier

High Point University

Grady Wright

Boise State University

This document was originally published by Society for Industrial and Applied Mathematics (SIAM) in SIAM Journal on Numerical Analysis. Copyright restrictions may apply. DOI: 10.1137/080730901 


\title{
STABILITY AND ERROR ESTIMATES FOR VECTOR FIELD INTERPOLATION AND DECOMPOSITION ON THE SPHERE WITH RBFS*
}

\author{
EDWARD J. FUSELIER ${ }^{\dagger}$ AND GRADY B. WRIGHT ${ }^{\ddagger}$
}

\begin{abstract}
A new numerical technique based on radial basis functions (RBFs) is presented for fitting a vector field tangent to the sphere, $\mathbb{S}^{2}$, from samples of the field at "scattered" locations on $\mathbb{S}^{2}$. The method naturally provides a way to decompose the reconstructed field into its individual Helmholtz-Hodge components, i.e., into divergence-free and curl-free parts, which is useful in many applications from the atmospheric and oceanic sciences (e.g., in diagnosing the horizontal wind and ocean currents). Several approximation results for the method will be derived. In particular, Sobolevtype error estimates are obtained for both the interpolant and its decomposition. Optimal stability estimates for the associated interpolation matrices are also presented. Finally, numerical validation of the theoretical results is given for vector fields with characteristics similar to those of atmospheric wind fields.
\end{abstract}

Key words. sphere, vector field decomposition, mesh-free, divergence-free, curl-free, radial basis functions, numerical modeling

AMS subject classifications. 41A05, 41A63, 41A30, 76M25, 86-08, 86A10

DOI. $10.1137 / 080730901$

1. Introduction. Vector fields tangent to the surface of the sphere $\mathbb{S}^{2}$ appear in many applications. For example, in the atmospheric sciences the horizontal velocity of the air in the atmosphere (horizontal wind field) is modeled as a tangent vector field, while the same is true in the oceanic sciences for the horizontal velocity of the water in the oceans (surface ocean currents). According to the Helmholtz-Hodge decomposition, any vector field can be decomposed into three components: a divergence-free component, a curl-free component, and a harmonic component, which is both curland divergence-free. It is well-known that a vector field cannot be simultaneously tangent to and harmonic on $\mathbb{S}^{2}$, which ultimately allows every tangent vector field to be decomposed uniquely into divergence-free and curl-free components. This decomposition can often provide useful diagnostic information in applications. For example, in the atmosphere the divergence-free (or rotational) part of the horizontal wind field gives details about cyclonic storms, while the curl-free (or irrotational) part gives details on high and low pressure systems [17, Ch. 3-4]. Similarly, in the ocean, these respective components of the horizontal ocean currents give information on gyres and overturning flow [36].

We introduce a new technique using radial basis functions (RBFs) for approximating a tangent vector field and its individual Helmholtz-Hodge components from samples of the field at "scattered" locations on $\mathbb{S}^{2}$. This is important, since often the values of these vector fields may be known only at nongridded locations, e.g., from measurement taken from rawinsondes, airplanes, buoys, remote sensing devices, or

${ }^{*}$ Received by the editors July 23, 2008; accepted for publication (in revised form) July 6, 2009; published electronically October 9, 2009.

http://www.siam.org/journals/sinum/47-5/73090.html

${ }^{\dagger}$ Department of Mathematics and Computer Science, High Point University, High Point, NC 27262-3598 (efuselie@highpoint.edu).

${ }^{\ddagger}$ Department of Mathematics, Boise State University, Boise, ID 83725-1555 (wright@math. boisestate.edu). This author's research was supported by National Science Foundation grant ATM0801309. 
from output from certain numerical models (cf. [39, section 4]). Unlike many of the current methods for accomplishing this same task (cf. [20, Ch. 4] and [22]) which are based on approximating the stream function (divergence-free part) and velocity potential (curl-free part) of the field, our method requires no grid, no computation of the divergence and vorticity of the field, and no coordinate singularities. Furthermore, the components of the field can be evaluated at any location on the sphere. The downside, however, is that it may be more computationally expensive.

Techniques similar to the one presented here for divergence-free and curl-free approximation on $\mathbb{S}^{2}$ were done in the early 1980's by Wahba [35]. There the author used vector spherical harmonics, which yield an approximation of the target field that can be decomposed into its divergence-free and curl-free components with little difficulty. Later, Freeden and Gervens [8] considered a similar approximation technique using vector spherical splines. The authors introduced positive definite kernels for fitting and decomposing a field using spherical basis functions (SBFs). Further progress in the area of vectorial approximation on the sphere via kernels has been made by the Geomathematics Group at the University of Kaiserslautern $[1,5,10]$. In none of these methods, however, were optimal error estimates or stability results obtained. Furthermore, these methods are intrinsic to the sphere and do not extend to general manifolds.

The present technique is based on the recent work of Narcowich, Ward, and Wright [32] in which a new class of positive definite kernels based on RBFs was introduced that yields divergence-free approximants to tangent vector fields on $\mathbb{S}^{2}$ (and general manifolds). For the case that these divergence-free approximants interpolate the data, stability and Sobolev error estimates have since been given [13]. We use a similar methodology and introduce a kernel from which one can build curl-free approximants and a "full" kernel that can be used for fitting and decomposing a vector field into its divergence-free and curl-free components. Following [13], we will present stability estimates for the interpolation matrices associated with these new kernels. Further, for the full kernel we will present error estimates for both the interpolant and its vector decomposition. We note that two more novelties of this technique are that the kernels used for fitting the data can be easily constructed from standard RBFs, and the method can be generalized to fitting vector fields tangent to general manifolds.

Before we introduce how to construct the decomposition, we first explain how to construct an interpolant to a tangent vector field on $\mathbb{S}^{2}$ given a kernel $\Psi(x, y)$. We use extrinsic (Cartesian) coordinates since they suffer no pole singularities. Let $x, y \in \mathbb{S}^{2}$, and $\Psi(x, y)$ be a $3 \times 3$ matrix-valued function with the following property: if $\mathbf{s}$ is tangent to $\mathbb{S}^{2}$ at a point $y$, then $\Psi(x, y)$ s defines a tangent vector field at $x$. Given nodes $X=\left\{x_{j}\right\}_{j=1}^{N} \subset \mathbb{S}^{2}$ and a tangent vector field $\mathbf{f}$ sampled on $X$, we look for an interpolant of the form

$$
\mathbf{t}(x)=\sum_{j=1}^{N} \Psi\left(x, x_{j}\right) \mathbf{s}_{j},
$$

where $\mathbf{s}_{j}$ is tangent to $\mathbb{S}^{2}$ at $x_{j}$. Finding the coefficient vectors amounts to solving the following linear system:

$$
\sum_{j=1}^{N} \Psi\left(x_{i}, x_{j}\right) \mathbf{s}_{j}=\mathbf{f}\left(x_{i}\right), \quad 1 \leq i \leq N .
$$

If $\Psi$ is positive definite, then the matrix associated with this linear system will be 
positive definite for any distinct point set $X$, and hence invertible.

As mentioned above, Narcowich, Ward, and Wright [32] introduced positive definite, divergence-free kernels on $\mathbb{S}^{2}$, which we denote by $\Psi_{\text {div }}$. Using a similar approach, we will derive positive definite, curl-free kernels $\Psi_{\text {curl }}$ on $\mathbb{S}^{2}$. The idea for constructing kernels $\Psi$ that can be used to fit and decompose a vector field is then to let $\Psi:=\Psi_{\text {div }}+\Psi_{\text {curl }}$. Since the sum of two positive definite functions is again positive definite, $\Psi$ is also positive definite. Using this kernel in (1), the Helmholtz-Hodge decomposition of the interpolant $\mathbf{t}$ is simply

$$
\mathbf{t}(x)=\sum_{j=1}^{N} \Psi\left(x, x_{j}\right) \mathbf{s}_{j}=\underbrace{\sum_{j=1}^{N} \Psi_{\text {div }}\left(x, x_{j}\right) \mathbf{s}_{j}}_{\text {Div.-free }}+\underbrace{\sum_{j=1}^{N} \Psi_{\text {curl }}\left(x, x_{j}\right) \mathbf{s}_{j}}_{\text {Curl-free }} .
$$

The main goals of this paper are to demonstrate that $\mathbf{t}$ not only approximates the field being interpolated, but that the terms in the decomposition of $\mathbf{t}$ approximate the appropriate divergence-free and curl-free parts of the target field.

While the applications discussed so far have been on the decomposition of vector fields, we also want to mention a few applications pertaining to just the approximation of a tangent vector field in which this new technique could potentially be used. If the kernel $\Psi$ is smooth enough, then the interpolant (1) can be analytically differentiated to provide an approximation to the divergence or vorticity of the target field. These quantities are important in meteorology since they are used as the prognostic variables in many of the models for numerical weather prediction [16, pp. 26-28]. To initialize these models for a forecast (also called spin-up), an initial value for the vorticity and divergence is needed. This involves several steps, one of which is using estimates of these values from observed wind data (cf. [3]). Another application pertains to the shallow water wave equations on a rotating sphere, which describe the motion of a fluid in a single hydrostatic layer and are used not only as a simplified model for the horizontal dynamics of the atmosphere (the "dynamic core") [40], but also as a model for tidal motion [23]. The velocity field of the fluid in these models is a tangent vector field. The approximation technique introduced in this paper could be used to fit the velocity fields generated from simulations of these models, or directly used in the simulation as the representation of the velocity fields, as done for scalar RBF approximation in [6].

The paper is organized as follows. In section 2 we introduce the necessary background and notation. The construction of the vector SBF interpolant is given in detail in section 3 . We give optimal stability estimates for the interpolation process in section 4. Various error estimates are given in section 5, including the main result of the paper, which is to show that the vector SBF interpolant simultaneously approximates each term in the Helmholtz-Hodge decomposition of the underlying target function. Also, we note that error estimates will be derived when the target function is too rough to be in the native space of the SBF kernel. Finally, we end the paper by verifying our results with physically relevant numerical examples.

2. Notation and preliminaries. We will use the following convention for the Fourier transform of a function or tempered distribution $f$ on $\mathbb{R}^{n}$ :

$$
\widehat{f}(\xi):=\int_{\mathbb{R}^{n}} f(x) e^{-i x^{T} \xi} d x .
$$


If $M$ is a manifold and $x \in M$, we denote the collection of vectors tangent to $M$ at $x$ by $T_{x} M$. We write elements of $T_{x} M$ in boldface. We will also denote vector functions in boldface, and the space of continuous tangent vector fields on $M$ will be given by $T M$. Sobolev spaces on $\mathbb{R}^{n}$ will play a role in the discussion that follows; we will follow the notation of [2]. We use the usual $L_{2}$-inner product for scalar functions on the sphere, namely if $f$ and $g$ are in $L_{2}\left(\mathbb{S}^{2}\right)$, then their inner product is given by

$$
\langle f, g\rangle=\int_{\mathbb{S}^{2}} f(x) g(x) d \mu(x),
$$

where $d \mu(x)$ is the surface measure on the sphere. If $\mathbf{f}$ and $\mathbf{g}$ are in $T \mathbb{S}^{2}$, we define their inner product by

$$
\langle\mathbf{f}, \mathbf{g}\rangle=\int_{\mathbb{S}^{2}} \mathbf{f}(x)^{T} \mathbf{g}(x) d \mu(x),
$$

where the dot product is taken in local coordinates. The closure of $T \mathbb{S}^{2}$ in this inner product will also be denoted by $L_{2}\left(\mathbb{S}^{2}\right)$. This should cause no confusion.

In what follows it will be necessary to use tangential differential operators on the sphere. We will let $\nabla_{*}$ denote the surface-gradient, let $\mathbf{L}$ be the surface-curl, and let $\Delta$ be the surface-Laplacian. It is not hard to show that $\nabla_{*}^{T} \nabla_{*}=\mathbf{L}^{T} \mathbf{L}=-\Delta$. Further, a surface-curl of a scalar function is divergence-free, and fields that are gradients of scalar functions are curl-free.

The geodesic distance between two points $x, y \in \mathbb{S}^{2}$ will be denoted by $d(x, y)$. In what follows error and stability estimates will be given in terms of the separation distance $q_{X}$ and the mesh norm $h_{X}$ of a finite point set $X=\left\{x_{1}, \ldots, x_{N}\right\} \subset \mathbb{S}^{2}$. These are defined by

$$
q_{X}:=\frac{1}{2} \min _{i \neq j} d\left(x_{i}, x_{j}\right) \quad \text { and } \quad h_{X}:=\sup _{x \in \mathbb{S}^{2}} \min _{x_{j} \in X} d\left(x, x_{j}\right) .
$$

We will also need similar quantities on $\mathbb{R}^{n}$. Let $\Omega \subset \mathbb{R}^{n}$ be bounded, and let $X \subset \mathbb{R}^{n}$ be a finite set of distinct points. We denote the separation radius and mesh norm in this case as

$$
q_{X, \mathbb{R}^{n}}:=\frac{1}{2} \min _{i \neq j}\left\|x_{i}-x_{j}\right\|_{2} \quad \text { and } \quad h_{X, \Omega}:=\sup _{x \in \Omega} \min _{x_{j} \in X}\left\|x-x_{j}\right\|_{2} .
$$

2.1. Scalar function spaces on $\mathbb{S}^{2}$. A good understanding of functions on the sphere requires one to be well-versed in spherical harmonics. The standard reference for spherical harmonics is Müller's book [26]. We let $\left\{Y_{\ell, m} \mid-\ell \leq m \leq \ell\right\}$ denote the spherical harmonics of degree $\ell$ on $\mathbb{S}^{2}$. These form an orthonormal basis of the eigenspace corresponding to the eigenvalue $\ell(\ell+1)$ of the Laplace-Beltrami operator $\Delta$ on the sphere. Every function in $L_{2}\left(\mathbb{S}^{2}\right)$ has a Fourier representation of the form

$$
f=\sum_{\ell=0}^{\infty} \sum_{m=-\ell}^{\ell} \widehat{f}(\ell, m) Y_{\ell, m}(x) \quad \text { with } \quad \widehat{f}(\ell, m)=\left\langle f, Y_{\ell, m}\right\rangle .
$$

The reader will note that we have used $\widehat{f}$ to denote the Fourier transform for functions on the sphere and on $\mathbb{R}^{n}$; the meaning of this notation will be made clear by its context. 
From this representation we also have Sobolev functions, whose norms can be given via

$$
\|f\|_{H^{\tau}\left(\mathbb{S}^{2}\right)}^{2}=\sum_{\ell=0}^{\infty} \sum_{m=-\ell}^{\ell}(1+\ell(\ell+1))^{\tau}|\widehat{f}(\ell, m)|^{2} .
$$

Sobolev spaces on the sphere, or any other manifold, can also be given in terms of charts. Let $\left\{U_{j}, \psi_{j}\right\}_{j=1}^{N}$ be an atlas of charts for a manifold $M$ of dimension $n$. For every such atlas there is a collection $\left\{\chi_{j}: M \rightarrow \mathbb{R}\right\}_{j=1}^{N}$ of $C^{\infty}$ functions that satisfy

$$
\chi_{j} \geq 0, \quad \operatorname{supp}\left(\chi_{j}\right) \subseteq U_{j}, \quad \sum_{1}^{N} \chi_{j}=1 \text { on } M .
$$

Also, for $f: M \rightarrow \mathbb{R}$ we define the projections $\pi_{j}(f): \mathbb{R}^{n} \rightarrow \mathbb{R}$ by

$$
\pi_{j}\left(\chi_{j} f\right)=\left\{\begin{array}{cl}
\chi_{j} f\left(\psi^{-1}(x)\right), & x \in B(0,1) \\
0 & \text { otherwise }
\end{array}\right.
$$

where $B(0,1)$ is the unit ball in $\mathbb{R}^{n}$. The Sobolev space $W_{p}^{\tau}(M)$ can then be defined by those functions whose projections are in $W_{p}^{\tau}\left(\mathbb{R}^{n}\right)$. The norm for this space is defined by

$$
\|f\|_{W_{p}^{\tau}(M)}=\left(\sum_{j=1}^{N}\left\|\pi_{j}\left(\chi_{j} f\right)\right\|_{W_{p}^{\tau}(M)}^{2}\right)^{1 / 2} .
$$

These spaces are independent of the choice of charts, and when different charts are used the norms are equivalent [21].

2.2. Tangent vector fields on $\mathbb{S}^{2}$. There is a vectorial analogue of spherical harmonics called vector-spherical harmonics $[8,9]$. They are not as well-known as scalar spherical harmonics, but they are widely used when one is dealing with geophysical applications that are vectorial in nature. These functions come in three $L_{2}$-orthogonal types: two types that are tangent to the sphere and one that is normal to the sphere. We are interested in the tangent fields, which are neatly separated into divergence-free and curl-free fields.

The divergence-free vector-spherical harmonics are given by

$$
\mathbf{y}_{\ell, m}=\mathbf{L} Y_{\ell, m} / \sqrt{\ell(\ell+1)},
$$

provided, of course, that $\ell \neq 0$. These are clearly divergence-free, and a quick application of integration by parts shows that they are orthonormal in $L_{2}(\mathbb{S})$. Similarly, one has curl-free vector spherical harmonics given by

$$
\mathbf{z}_{\ell, m}=\nabla_{*} Y_{\ell, m} / \sqrt{\ell(\ell+1)} .
$$

These divergence-free and curl-free spherical harmonics are precisely the eigenfunctions for the Laplace-Beltrami operator operating on tangent vector fields, and they form a complete orthonormal set for $L_{2}\left(\mathbb{S}^{2}\right)$. We will be interested in several function spaces generated by these fields, which we define now as follows:

$$
\boldsymbol{\Sigma}_{\ell, \text { div }}:=\operatorname{span}\left\{\mathbf{y}_{\ell, m} \mid-\ell \leq m \leq \ell\right\}, \quad \boldsymbol{\Sigma}_{\ell, \text { curl }}:=\operatorname{span}\left\{\mathbf{z}_{\ell, m} \mid-\ell \leq m \leq \ell\right\},
$$




$$
\Sigma_{L}:=\bigoplus_{\ell=1}^{L}\left(\Sigma_{\ell, \text { div }} \bigoplus \Sigma_{\ell, \text { curl }}\right)
$$

Vector-spherical harmonics give a Fourier analysis on $L_{2}\left(\mathbb{S}^{2}\right)$ vector fields. We will denote the Fourier symbols for a vector function $\mathbf{f}$ as

$$
\widetilde{\mathbf{f}}_{d i v}(\ell, m):=\left\langle\mathbf{f}, \mathbf{y}_{\ell, m}\right\rangle \quad \text { and } \quad \widetilde{\mathbf{f}}_{c u r l}(\ell, m):=\left\langle\mathbf{f}, \mathbf{z}_{\ell, m}\right\rangle .
$$

Each vector function $\mathbf{f} \in L_{2}\left(\mathbb{S}^{2}\right)$ has the Fourier expansion

$$
\mathbf{f}=\sum_{\ell=1}^{\infty} \sum_{m=-\ell}^{\ell}\left(\widetilde{\mathbf{f}}_{d i v}(\ell, m) \mathbf{y}_{\ell, m}+\widetilde{\mathbf{f}}_{c u r l}(\ell, m) \mathbf{z}_{\ell, m}\right) .
$$

Inner products are then given by

$$
\langle\mathbf{f}, \mathbf{g}\rangle=\sum_{\ell=1}^{\infty} \sum_{m=-\ell}^{\ell}\left(\widetilde{\mathbf{f}}_{d i v}(\ell, m) \widetilde{\mathbf{g}}_{d i v}(\ell, m)+\widetilde{\mathbf{f}}_{c u r l}(\ell, m) \widetilde{\mathbf{g}}_{c u r l}(\ell, m)\right) .
$$

With this we have the vectorial Sobolev space $H^{\tau}\left(\mathbb{S}^{2}\right)$, which is the set of all $\mathbf{f} \in L_{2}\left(\mathbb{S}^{2}\right)$ such that

$$
\|\mathbf{f}\|_{H^{\tau}\left(\mathbb{S}^{2}\right)}^{2}=\sum_{\ell=1}^{\infty} \sum_{m=-\ell}^{\ell}(1+\ell(\ell+1))^{\tau}\left(\left|\widetilde{\mathbf{f}}_{d i v}(\ell, m)\right|^{2}+\left.\widetilde{\mathbf{f}}_{\text {curl }}(\ell, m)\right|^{2}\right)<\infty .
$$

Like the scalar case, we will denote this space by $H^{\tau}\left(\mathbb{S}^{2}\right)$. It will be clear which space we mean by its context. We denote by $H_{\text {div }}^{\tau}\left(\mathbb{S}^{2}\right)$ and $H_{\text {curl }}^{\tau}\left(\mathbb{S}^{2}\right)$ the divergence-free and curl-free subspaces of $H^{\tau}\left(\mathbb{S}^{2}\right)$, respectively. As with scalar functions, one can also define Sobolev spaces for tangent vector fields in terms of charts, with norms equivalent to those given. The only complication is that locally one has to deal with vector-valued functions instead of scalar-valued functions [14].

2.3. Positive definite functions on the sphere. An important class of positive definite functions on the sphere are SBFs [38, Ch. 17]. These are scalar-valued zonal functions with positive Fourier coefficients, i.e., their Fourier series are of the form

$$
\psi(x \cdot y)=\sum_{\ell=0}^{\infty} \widehat{\psi}(\ell) \sum_{m=-\ell}^{\ell} Y_{\ell, m}(x) Y_{\ell, m}(y),
$$

where $\widehat{\psi}(\ell)>0$ for all $\ell$. In the following sections we illustrate how these can be used to construct other useful positive definite functions.

We are ultimately interested in tangent kernels on $\mathbb{S}^{2}$, but it is just as easy to define these kernels on general manifolds. Let $M$ be an $n$-dimensional manifold. The kernel $\Psi(x, y)$, locally given by an $n \times n$ matrix-valued function from $M \times M$ to $\mathbb{R}^{n} \times \mathbb{R}^{n}$, is called positive semidefinite if we have

$$
\sum_{j, k} \mathbf{s}_{k}^{T} \Psi\left(x_{k}, x_{j}\right) \mathbf{s}_{j} \geq 0
$$

for all finite point sets $X=\left\{x_{j}\right\}_{j=1}^{N} \subset M$ and all $\mathbf{s}_{j} \in T_{x_{j}} M$. When the above quadratic form being zero implies that $\mathbf{s}_{j}=\mathbf{0}$ for all $j$, we say that $\Psi$ is positive 
definite. We will be interested in positive definite tangent kernels which have the additional property that $\Psi\left(x, x_{j}\right) \mathbf{s}_{j} \in T M$ provided $\mathbf{s}_{j} \in T_{x_{j}} M$.

Recently, positive definite kernels on the sphere were introduced that yield interpolants with no surface divergence [32]. As it turns out they are relatively easy to construct. Given an SBF $\psi$, we define the function $\Psi_{d i v}$ via

$$
\Psi_{d i v}(x, y):=\mathbf{L}_{x} \mathbf{L}_{y}^{T}[\psi(x \cdot y)],
$$

where $\mathbf{L}_{x}$ and $\mathbf{L}_{y}^{T}$ act only on the $x$ and $y$ variables, respectively. This kernel is positive definite and gives rise to divergence-free interpolants on the sphere. To see that $\Psi_{d i v}$ is positive semidefinite, one first expands $\psi$ in a Fourier series to get

$$
\begin{aligned}
\Psi_{d i v} & =\mathbf{L}_{x} \mathbf{L}_{y}^{T}\left(\sum_{\ell=0}^{\infty} \sum_{m=-\ell}^{\ell} \widehat{\psi}(\ell) Y_{\ell, m}(x) Y_{\ell, m}(y)\right)=\sum_{\ell=1}^{\infty} \sum_{m=-\ell}^{\ell} \widehat{\psi}(\ell) \mathbf{L}_{x} Y_{\ell, m}(x) \mathbf{L}_{y}^{T} Y_{\ell, m}(y) \\
& =\sum_{\ell=1}^{\infty} \ell(\ell+1) \widehat{\psi}(\ell) \sum_{m=-\ell}^{\ell} \mathbf{y}_{\ell, m}(x) \mathbf{y}_{\ell, m}^{T}(y) .
\end{aligned}
$$

Here we require that $\widehat{\psi}(\ell)=\mathcal{O}\left(\ell^{-4-\epsilon}\right)$ for some $\epsilon>0$, which ensures that the above series is convergent and continuous in both variables. Given a discrete set of points $X \subset \mathbb{S}^{2}$ with corresponding tangent vectors $\mathbf{s}_{j} \in T_{x_{j}} \mathbb{S}^{2}$, we have

$$
\begin{aligned}
\sum_{j, k=1}^{N} \mathbf{s}_{k}^{T} \Psi_{d i v}\left(x_{k}, x_{j}\right) \mathbf{s}_{j} & =\sum_{j, k} \sum_{\ell=1}^{\infty} \ell(\ell+1) \widehat{\psi}(\ell) \sum_{m=-\ell}^{\ell} \mathbf{s}_{k}^{T} \mathbf{y}_{\ell, m}\left(x_{k}\right) \mathbf{y}_{\ell, m}^{T}\left(x_{j}\right) \mathbf{s}_{j} \\
& =\sum_{\ell=1}^{\infty} \ell(\ell+1) \widehat{\psi}(\ell) \sum_{m=-\ell}^{\ell}\left|\sum_{j=1}^{N} \mathbf{s}_{k}^{T} \mathbf{y}_{\ell, m}\left(x_{j}\right)\right|^{2} \geq 0 .
\end{aligned}
$$

Further, if $\widehat{\psi}(\ell)>0$ for all $\ell \geq 1$, then $\Psi_{\text {div }}$ is positive definite. For a proof, see [32, section 2.2.1].

Naturally, there is a curl-free analogue that we discuss now. Recall that $\mathbf{f}$ is curlfree if and only if $\mathbf{f}=\nabla_{*} f$ for some scalar potential $f$. Motivated by this, given an SBF $\psi$, we can construct the matrix-valued kernel

$$
\Psi_{\text {curl }}(x, y):=\nabla_{* x} \nabla_{* y}^{T}[\psi(x \cdot y)] .
$$

Again, if $\widehat{\psi}(\ell)>0$ for all $\ell \geq 1$, then $\Psi_{\text {curl }}$ is positive definite. The proof is the same as the divergence-free case, with the only modification being to change all occurrences of $\mathbf{y}_{\ell, m}$ to $\mathbf{z}_{\ell, m}$. Also, note that the sum of positive definite functions is again positive definite. As alluded to in the introduction, in what follows we will also be interested in the positive definite kernel

$$
\Psi:=\Psi_{d i v}+\Psi_{c u r l} .
$$

2.4. Constructing kernels on $\mathbb{S}^{2}$ from $R B F s$ in $\mathbb{R}^{3}$. Above we gave a construction of divergence-free and curl-free kernels from SBFs. However, it is possible to construct them out of RBFs on $\mathbb{R}^{3}$. In fact, this is how divergence-free SBFs were derived in [32]. The ability to do this follows from the fact that an $\mathrm{RBF}$ restricted to the sphere gives a zonal SBF. Indeed, if $x, y \in \mathbb{S}^{2}$, then we have 
$\phi(x, y)=\phi\left(\|x-y\|_{2}\right)=\phi(\sqrt{2-2(x \cdot y)}):=\psi(x \cdot y)$. In what follows we will always let $\psi(x, y)$ denote the SBF obtained by restricting the $\operatorname{RBF} \phi(x, y)$ to the sphere.

Let $x \in \mathbb{S}^{2}$, and let $\mathbf{n}_{x}$ denote the unit normal vector to $\mathbb{S}^{2}$ at $x$. When viewed as acting on vector fields in $\mathbb{R}^{3}$, the operator $\mathbf{L}=\mathbf{n}_{x} \times \nabla_{*}$ becomes $Q_{x} \nabla$, where $\nabla$ is the usual gradient on $\mathbb{R}^{3}$, and $Q_{x}$ is the matrix such that $Q_{x} \mathbf{s}=\mathbf{n}_{x} \times \mathbf{s}$, i.e.,

$$
Q_{x}:=\left(\begin{array}{ccc}
0 & -x_{3} & x_{2} \\
x_{3} & 0 & -x_{1} \\
-x_{2} & x_{1} & 0
\end{array}\right) .
$$

Using this, we get the following formula for $\Psi_{d i v}$ :

$$
\Psi_{d i v}(x, y)=Q_{x}^{T}\left(-\nabla \nabla^{T} \phi(x, y)\right) Q_{y} .
$$

Similarly, the surface gradient can be written as $\nabla_{*}=P_{x} \nabla$, where $P_{x}=I-x x^{T}$ projects vectors onto $T_{x} \mathbb{S}^{2}$. This gives us

$$
\Psi_{\text {curl }}(x, y)=P_{x}^{T}\left(-\nabla \nabla^{T} \phi(x, y)\right) P_{y} .
$$

Note the relationship of $\Psi_{c u r l}$ and $\Psi_{d i v}$ in (7) and (8) to the matrix-valued function $\Phi_{\text {curl }}:=-\nabla \nabla^{T} \phi(x, y)$. The function $\Phi_{\text {curl }}$ is positive definite and gives curl-free interpolants on $\mathbb{R}^{3}[11,12]$.

In what follows we will rely on results already known for $\Phi_{\text {curl }}$ to aid in our discussion of $\Psi_{d i v}, \Psi_{\text {curl }}$, and $\Psi$.

2.5. Native spaces. It is well-known that every positive definite function $\psi$ naturally gives rise to a reproducing kernel Hilbert space, usually referred to as the native space of $\psi$ and denoted by $\mathcal{N}_{\psi}$. Approximation via linear shifts of $\psi$ is wellunderstood within this space, so a thorough knowledge of $\mathcal{N}_{\psi}$ is important. For SBFs, the native space has the following characterization:

$$
\mathcal{N}_{\psi}=\left\{f \in L_{2}\left(\mathbb{S}^{2}\right) \mid \sum_{\ell=0}^{\infty} \sum_{m=-\ell}^{\ell} \frac{|\widehat{f}(\ell, m)|^{2}}{\widehat{\psi}(\ell)}<\infty\right\},
$$

with inner product given by

$$
\langle f, g\rangle_{\mathcal{N}_{\psi}}=\sum_{\ell=0}^{\infty} \sum_{m=-\ell}^{\ell} \frac{\widehat{f}(\ell, m) \widehat{g}(\ell, m)}{\widehat{\psi}(\ell)} .
$$

This is useful for us because it allows us to work on familiar function spaces, such as Sobolev spaces. Indeed, if $\widehat{\psi}(\ell) \sim(1+\ell(\ell+1))^{-\tau}$, then it follows that $\mathcal{N}_{\psi}=H^{\tau}\left(\mathbb{S}^{2}\right)$ with equivalent norms.

In the case of tangent kernels, we define the native space as follows. Let $\mathcal{F}$ be a Hilbert space of vector-valued functions tangent to an $n$-dimensional manifold $M$. A continuous function $\Psi$, locally given by an $n \times n$ matrix-valued function, is called a reproducing kernel for $\mathcal{F}$ if for all $x \in M$ and $\mathbf{s} \in T_{x} M$ we have the following:

1. $\Psi(\cdot, x) \mathbf{s} \in \mathcal{F}$.

2. $\mathbf{s}^{T} \mathbf{f}(x)=\langle\mathbf{f}, \Psi(\cdot, x) \mathbf{s}\rangle_{\mathcal{F}}$ for all $\mathbf{f} \in \mathcal{F}$.

If $\mathcal{F}$ and $\Psi$ satisfy the above properties, we say $\mathcal{F}$ is the native space for the kernel $\Psi$, and we denote $\mathcal{F}$ by $\mathcal{N}_{\Psi}$. 
Interpolants generated by the reproducing kernel enjoy nice properties within the natives space. In particular, if $\mathbf{f}$ is in $\mathcal{N}_{\Psi}$ and $I_{X} \mathbf{f}$ is the interpolant to $\mathbf{f}$ generated by the kernel $\Psi$, then one gets the Pythagorean law

$$
\left\|\mathbf{f}-I_{X} \mathbf{f}\right\|_{\mathcal{N}_{\Psi}}^{2}+\left\|I_{X} \mathbf{f}\right\|_{\mathcal{N}_{\Psi}}^{2}=\|\mathbf{f}\|_{\mathcal{N}_{\Psi}}^{2} .
$$

This immediately gives us the following estimate:

$$
\left\|\mathbf{f}-I_{X} \mathbf{f}\right\|_{\mathcal{N}_{\Psi}} \leq\|\mathbf{f}\|_{\mathcal{N}_{\Psi}} .
$$

It is useful when the native space can be characterized as a concrete function space. In [13, Theorem 2.2] it was shown that the inner product for $\mathcal{N}_{\Psi_{d i v}}$ is

$$
\langle\mathbf{f}, \mathbf{g}\rangle_{\mathcal{N}_{\Psi_{d i v}}}=\sum_{\ell=1}^{\infty} \sum_{m=-\ell}^{\ell} \frac{\widetilde{\mathbf{f}}_{d i v}(\ell, m) \widetilde{\mathbf{g}}_{d i v}(\ell, m)}{\ell(\ell+1) \widehat{\psi}(\ell)},
$$

giving

$$
\mathcal{N}_{\Psi_{d i v}}=\left\{\mathbf{f} \in H_{d i v}^{0}\left(\mathbb{S}^{2}\right) \mid \sum_{\ell=1}^{\infty} \sum_{m=-\ell}^{\ell} \frac{\left.\widetilde{\mathbf{f}}_{d i v}(\ell, m)\right|^{2}}{\ell(\ell+1) \widehat{\psi}(\ell)}<\infty\right\} .
$$

From this it follows that if $\widehat{\psi}(\ell) \sim(1+\ell(\ell+1))^{-(\tau+1)}$, then $\mathcal{N}_{\Psi_{d i v}}=H_{d i v}^{\tau}\left(\mathbb{S}^{2}\right)$ with equivalent norms.

There are similar characterizations of native spaces for $\Psi_{\text {curl }}$ and $\Psi$. Namely, $\mathcal{N}_{\Psi_{\text {curl }}}$ consists of all functions in $H_{\text {curl }}^{0}\left(\mathbb{S}^{2}\right)$ with finite norm in the inner product given by

$$
\langle\mathbf{f}, \mathbf{g}\rangle_{\mathcal{N}_{\Psi_{c u r l}}}=\sum_{\ell=1}^{\infty} \sum_{m=-\ell}^{\ell} \frac{\widetilde{\mathbf{f}}_{c u r l}(\ell, m) \widetilde{\mathbf{g}}_{c u r l}(\ell, m)}{\ell(\ell+1) \widehat{\psi}(\ell)} .
$$

The space $\mathcal{N}_{\Psi}$ is given by the closure of $L_{2}$ vector functions in the following inner product:

$$
\langle\mathbf{f}, \mathbf{g}\rangle_{\mathcal{N}_{\Psi}}=\sum_{\ell=1}^{\infty} \sum_{m=-\ell}^{\ell}\left(\frac{\widetilde{\mathbf{f}}_{c u r l}(\ell, m) \widetilde{\mathbf{g}}_{c u r l}(\ell, m)}{\ell(\ell+1) \widehat{\psi}(\ell)}+\frac{\widetilde{\mathbf{f}}_{d i v}(\ell, m) \widetilde{\mathbf{g}}_{d i v}(\ell, m)}{\ell(\ell+1) \widehat{\psi}(\ell)}\right) .
$$

As mentioned in the previous section, we will concentrate on kernels that are obtained from an RBF $\phi$. So that we can work with Sobolev functions, here and throughout the rest of the paper we will assume that the RBF $\phi$ satisfies

$$
\widehat{\phi}(\xi) \sim\left(1+\|\xi\|_{2}^{2}\right)^{-\left(\tau+\frac{3}{2}\right)},
$$

where $\tau>1$ and $\widehat{\phi}$ is the Fourier transform of $\phi$ in the $\mathbb{R}^{3}$ sense. We claim that this ensures that our native spaces are Sobolev. Indeed, if $\psi$ is an SBF obtained by restricting $\phi$ to the sphere, by [27, Proposition 4.1] we have that $\widehat{\psi}(\ell) \sim(1+$ $\ell(\ell+1))^{-(\tau+1)}$. The result is that if $\phi$ satisfies (10), we have $\mathcal{N}_{\Psi_{d i v}}=H_{\text {div }}^{\tau}\left(\mathbb{S}^{2}\right)$, $\mathcal{N}_{\Psi_{\text {curl }}}=H_{\text {curl }}^{\tau}\left(\mathbb{S}^{2}\right)$, and $\mathcal{N}_{\Psi}=H^{\tau}\left(\mathbb{S}^{2}\right)$, all with equivalent norms. We should mention that there are several well-known RBFs satisfying (10), such as Matérn [25] and Wendland [37] functions, which are both used for the numerical examples in section 6 . 
3. Constructing the vector SBF interpolants. The ability to use extrinsic coordinates for constructing the vector SBF interpolants has many benefits, with the primary one being that it entirely avoids any coordinate singularities introduced by the intrinsic coordinate system. For the sphere, these singularities are commonly called the "pole-problem." However, when using extrinsic coordinates one must be mindful that we are solving for tangent vectors. A naive approach to solving for the interpolation coefficient vectors might lead to a singular system of equations. Therefore we give a brief overview of how to set up the vector interpolant and the corresponding matrix for determining interpolation coefficients using the $\mathbb{R}^{3}$ coordinate system.

Let $X=\left\{x_{j}\right\}_{j=1}^{N}=\left\{\left(x_{j, 1}, x_{j, 2}, x_{j, 3}\right)\right\}_{j=1}^{N}$ be some distinct set of nodes on $\mathbb{S}^{2}$, and let $\left\{\mathbf{d}_{j}, \mathbf{e}_{j}, \mathbf{n}_{j}\right\}$ denote an orthonormal coordinate system at each $x_{j}$, where $\mathbf{n}_{j}$ is the outward normal to $\mathbb{S}^{2}, \mathbf{e}_{j}$ is a unit tangent vector, and $\mathbf{d}_{j}=\mathbf{n}_{j} \times \mathbf{e}_{j}$. The components for $\mathbf{n}_{j}$ are simply the coordinates of $x_{j}$, and obvious choices for the tangent vectors $\mathbf{d}_{j}$ and $\mathbf{e}_{j}$ are the standard meridional and zonal vectors, respectively:

$$
\mathbf{d}_{j}=\frac{1}{\sqrt{1-x_{j, 3}^{2}}}\left[\begin{array}{c}
-x_{j, 3} x_{j, 1} \\
-x_{j, 3} x_{j, 2} \\
1-x_{j, 3}^{2}
\end{array}\right], \quad \mathbf{e}_{j}=\frac{1}{\sqrt{1-x_{j, 3}^{2}}}\left[\begin{array}{c}
-x_{j, 2} \\
x_{j, 1} \\
0
\end{array}\right],
$$

which form an orthonormal basis for the tangent space $T_{x_{j}} \mathbb{S}^{2}$.

If $\mathbf{f}$ is some tangent vector field and $\left\{\mathbf{f}_{j}\right\}_{j=1}^{N}=\left\{\left[f_{j, 1} f_{j, 2} f_{j, 3}\right]^{T}\right\}_{j=1}^{N}$ are samples $\mathbf{f}$ on $X$, then the vector SBF interpolant to $\mathbf{f}$ is constructed from linear combinations of the tangent vector basis $\left\{\left(\Psi\left(x, x_{j}\right) \mathbf{d}_{j}, \Psi\left(x, x_{j}\right) \mathbf{e}_{j}\right)\right\}_{j=1}^{N}$, i.e., the interpolant is of the form

$$
I_{X} \mathbf{f}=\sum_{j=1}^{N} \Psi\left(x, x_{j}\right) \underbrace{\left[\alpha_{j} \mathbf{d}_{j}+\beta_{j} \mathbf{e}_{j}\right]}_{\mathbf{s}_{j}},
$$

where $\Psi$ is positive definite. Illustrations of the respective meridional and zonal basis vectors formed by $\Psi_{d i v}, \Psi_{\text {curl }}$, and $\Psi:=\Psi_{d i v}+\Psi_{\text {curl }}$ are displayed in Figure 1 .

The interpolation coefficient vectors $\mathbf{s}_{j}$ in (12) are determined by solving the linear system

$$
\sum_{j=1}^{N} \Psi\left(x_{i}, x_{j}\right)\left[\alpha_{j} \mathbf{d}_{j}+\beta_{j} \mathbf{e}_{j}\right]=\mathbf{f}_{i}, \quad 1 \leq i \leq N
$$

for $\alpha_{j}$ and $\beta_{j}$. However, this is not a square system since each $\mathbf{f}_{i}$ has three components. To make a square system, we note that $\mathbf{f}_{i}$ can also be expressed in terms of the orthonormal tangent vectors $\mathbf{d}_{i}$ and $\mathbf{e}_{i}$ as $\mathbf{f}_{i}=\gamma_{i} \mathbf{d}_{i}+\delta_{i} \mathbf{e}_{i}$, where

$$
\left[\begin{array}{c}
\gamma_{i} \\
\delta_{i}
\end{array}\right]=\left[\begin{array}{c}
\mathbf{d}_{i}^{T} \\
\mathbf{e}_{i}^{T}
\end{array}\right] \mathbf{f}_{i}
$$

Using this result, we can rewrite (13) as the $2 N \times 2 N$ linear system

$$
\sum_{j=1}^{N} \underbrace{\left(\left[\begin{array}{l}
\mathbf{d}_{i}^{T} \\
\mathbf{e}_{i}^{T}
\end{array}\right] \Psi\left(x_{i}, x_{j}\right)\left[\begin{array}{ll}
\mathbf{d}_{j} & \mathbf{e}_{j}
\end{array}\right]\right)}_{A_{i, j}^{(2)}}\left[\begin{array}{c}
\alpha_{j} \\
\beta_{j}
\end{array}\right]=\left[\begin{array}{l}
\gamma_{i} \\
\delta_{i}
\end{array}\right], \quad 1 \leq i \leq N .
$$




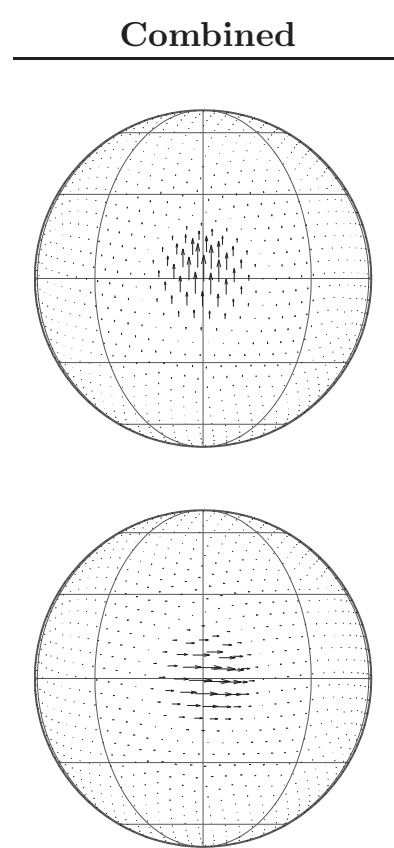

$=$

Div.-Free

$+$

Curl-Free

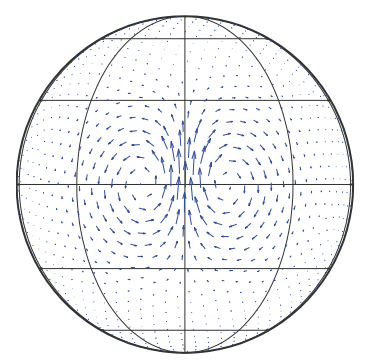

(a) Meridional basis $\Psi\left(x, x_{j}\right) \mathbf{d}_{j}$
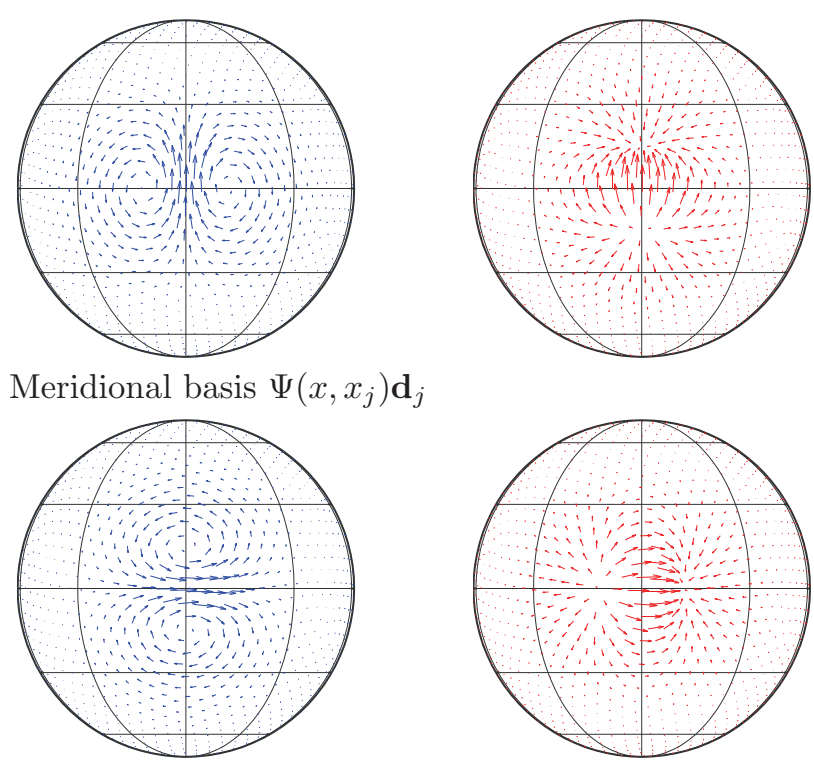

(b) Zonal basis $\Psi\left(x, x_{j}\right) \mathbf{e}_{j}$

FIG. 1. Illustration of the (a) meridional and (b) zonal vector basis functions formed by the tangent kernel $\Psi:=\Psi_{\text {div }}+\Psi_{\text {curl }}$ for interpolating and decomposing tangent vector fields. Here $x_{j}$ is chosen as $(1,0,0)$, and the vectors $\mathbf{d}_{j}$ and $\mathbf{e}_{j}$ are defined in (11). All the plots are orthographic projections of the fields displayed from $0^{\circ}$ longitude and $0^{\circ}$ latitude, and each field has been normalized by its max norm for displaying purposes. The Matérn RBF was used to construct $\Psi$.

The $(i, j)$ th $2 \times 2$ block of the $2 N \times 2 N$ matrix arising from this linear system is given by $A_{i, j}^{(2)}$. This interpolation matrix will be invertible if $\Psi$ is positive definite. In what follows, we will denote this interpolation matrix by $A_{X, \Psi}$ when $\Psi:=\Psi_{\text {div }}+\Psi_{\text {curl }}$, and $A_{X, \Psi_{\text {curl }}}$ when $\Psi:=\Psi_{\text {curl }}$.

We conclude by noting that if $\Psi$ is constructed from an $\operatorname{RBF} \phi$, then the following formula for $A_{i, j}^{(2)}$ can be worked out:

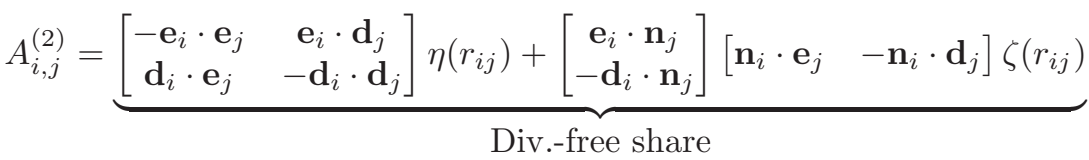

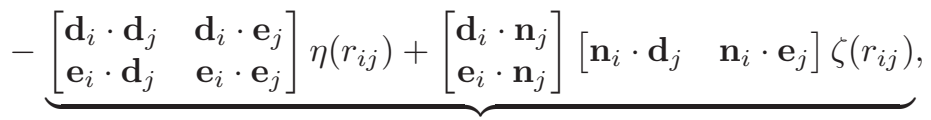

where $r_{i j}=\left\|x_{i}-x_{j}\right\|_{2}, \eta(r)=\phi^{\prime}(r) / r$, and $\zeta(r)=\eta^{\prime}(r) / r$.

4. Stability of the interpolation process. In this section we study the stability of the interpolation process when the tangent kernels $\Psi$ and $\Psi_{\text {curl }}$ are employed. We do this by first estimating the smallest eigenvalue of the interpolation matrix

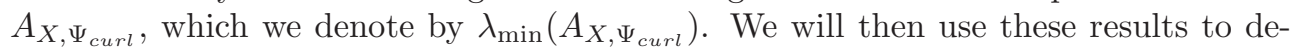
rive stability estimates for $A_{X, \Psi}$. As is typical in stability estimates for kernels, the estimates will depend upon the separation radius $q_{X}$ of the point set $X$ and the 
smoothness of the kernel. The reader should note that our arguments will be valid only when the matrix kernel is constructed from an $\mathrm{RBF}$ on $\mathbb{R}^{3}$.

Stability estimates for the divergence-free basis function $\Psi_{d i v}$ were given in $[13$, Theorem 3.8]. There the authors were able to use the form of the kernel given in (7) to "lift" the problem from the sphere to estimates involving the kernel $\Phi_{\text {curl }}$ in $\mathbb{R}^{3}$. More precisely, the authors were able to show that any eigenvalue of $A_{X, \Psi_{\text {div }}}$ is bounded from below by $\lambda_{\min }\left(A_{X, \Phi_{\text {curl }}}\right)$. They then used the following proposition.

Proposition 1 (see [11, Theorem 7]). Let $\phi$ be an even, positive definite function which possesses a positive Fourier transform $\widehat{\phi} \in C\left(\mathbb{R}^{n} / 0\right)$. With the function

$$
M(\sigma):=\inf _{\|\xi\|_{2} \leq \sigma} \widehat{\phi}(\xi)
$$

a lower bound on $\lambda_{\min }\left(A_{X, \Phi_{\text {curl }}}\right)$ is given by

$$
\lambda_{\min }\left(A_{X, \Phi_{\text {curl }}}\right) \geq\left(\frac{\sigma^{2}}{16 \pi}\right)^{(n+2) / 2} \frac{M(\sigma) \pi}{(4 \pi)^{n} \Gamma((n+2) / 2)}
$$

for any $\sigma>0$ satisfying

$$
\sigma \geq C / q_{X, \mathbb{R}^{n}}
$$

Here the constant $C$ is independent of $X$ and $\phi$.

Following [13], we will show that every eigenvalue of $A_{X, \Psi_{\text {curl }}}$ is also bounded from below by $\lambda_{\min }\left(A_{X, \Phi_{\text {curl }}}\right)$. We will transition from intrinsic to extrinsic coordinates on the sphere using the notation in section 3 . Let $\widetilde{\mathbf{c}}$ be a unit eigenvector of $A_{X, \Psi_{\text {curl }}}$ with corresponding eigenvalue $\lambda$. Let $\widetilde{\mathbf{c}}_{j}=\left[\alpha_{j} \beta_{j}\right]^{T}$ be the vector whose components are given by the $j$ th 2 -block of $\widetilde{\mathbf{c}}$. Finally we let $\mathbf{c}_{j}=\alpha_{j} \mathbf{d}_{j}+\beta_{j} \mathbf{e}_{j}$, which is the usual representation of $\widetilde{\mathbf{c}}_{j}$ in $\mathbb{R}^{3}$, and define $\mathbf{c}$ to be the $3 N \times 1$ vector whose $j$ th 3 -component block is given by $\mathbf{c}_{j}$. Now we have

$$
\lambda=\widetilde{\mathbf{c}}^{T} A_{X, \Psi_{\text {curl }}} \widetilde{\mathbf{c}}=\sum_{i, j} \widetilde{\mathbf{c}}_{i}^{T}\left[\begin{array}{c}
\mathbf{d}_{i}^{T} \\
\mathbf{e}_{i}^{T}
\end{array}\right] \Psi_{c u r l}\left(x_{i}, x_{j}\right)\left[\begin{array}{ll}
\mathbf{d}_{j} & \mathbf{e}_{j}
\end{array}\right] \widetilde{\mathbf{c}}_{j}=\sum_{i, j} \mathbf{c}_{i}^{T} \Psi_{c u r l}\left(x_{k}, x_{j}\right) \mathbf{c}_{j} .
$$

Rewriting $\Psi_{\text {curl }}$ in terms of $\Phi_{\text {curl }}$ as in (8), we continue to get

$$
\sum_{i, j} \mathbf{c}_{i}^{T}\left(P_{x_{i}}^{T} \Phi_{\text {curl }}\left(x_{i}, x_{j}\right) P_{x_{j}}\right) \mathbf{c}_{j}=\sum_{i, j} \mathbf{c}_{i}^{T} \Phi_{\text {curl }}\left(x_{i}, x_{j}\right) \mathbf{c}_{j} \geq \lambda_{\min }\left(A_{X, \Phi_{\text {curl }}}\right)\|\mathbf{c}\|_{2}^{2},
$$

where we have used the fact that $\mathbf{c}_{j} \in T_{x_{j}} \mathbb{S}^{2}$. Finally, note that $\|\mathbf{c}\|^{2}=\|\widetilde{\mathbf{c}}\|^{2}=1$. The result is $\lambda \geq \lambda_{\min }\left(A_{X, \Phi_{\text {curl }}}\right)$. With this estimate, Proposition 1 , and the fact that $q_{X, \mathbb{R}^{3}} \sim q_{X}$, we have the following theorem.

THEOREM 1. Let $\phi$ be an even positive definite function, which possesses a positive Fourier transform $\widehat{\phi} \in C\left(\mathbb{R}^{3} / 0\right)$. Let $\Psi_{\text {curl }}$ be the curl-free SBF kernel on $\mathbb{S}^{2}$ generated by $\phi$. With the function

$$
M(\sigma):=\inf _{\|\xi\|_{2} \leq \sigma} \widehat{\phi}(\xi),
$$

a lower bound on $\lambda_{\min }\left(A_{X, \Psi}\right)$ is given by

$$
\lambda_{\min }\left(A_{X, \Psi_{c u r l}}\right) \geq\left(\frac{\sigma^{2}}{16 \pi}\right)^{5 / 2} \frac{M(\sigma) \pi}{(4 \pi)^{3} \Gamma(5 / 2)}
$$

Copyright (C) by SIAM. Unauthorized reproduction of this article is prohibited. 
for any $\sigma>0$ satisfying

$$
\sigma \geq C / q_{X}
$$

Here the constant $C$ is independent of $X$ and $\phi$.

When the kernel has finite smoothness, i.e., $\phi$ satisfies (10), we get the following corollary.

Corollary 1. If the Fourier transform of the RBF $\phi$ satisfies (10), then the smallest eigenvalue of the interpolation matrix $A_{X, \Psi_{c u r l}}$ can be bounded by

$$
\lambda_{\min }\left(A_{X, \Psi_{\text {curl }}}\right) \geq C q_{X}^{2 \tau-2},
$$

where $C$ is a constant independent of $X$ and $\Psi_{\text {curl }}$.

It can be shown by a similar argument as in [13] that, in terms of the power of the separation radius, these estimates are the best possible. Also note that Theorem 1, when combined with the analogous divergence-free result in [13], automatically gives us stability estimates for $A_{X, \Psi}$, which we state below.

Corollary 2. If the Fourier transform of the RBF $\phi$ satisfies (10), then the smallest eigenvalue of the interpolation matrix $A_{X, \Psi}$ can be bounded by

$$
\lambda_{\min }\left(A_{X, \Psi}\right) \geq C q_{X}^{2 \tau-2},
$$

where $C$ is a constant independent of $X$ and $\Psi$.

5. Error rates. The purpose of this section is to demonstrate that vector SBF interpolants approximate a wide class of functions, including those outside of the native space. We begin the section with a study of interpolation and approximation via vector spherical polynomials. Then we proceed to derive approximation rates for functions within the native space. Using the standard "doubling trick" from splines, we will show next that smoother functions within the native space enjoy faster approximation rates. We then concentrate on target functions that are too rough to be in the native space. Finally, we will present the main result of the paper by showing that the approximation takes place simultaneously on each term of the Helmholtz-Hodge decomposition, i.e., the divergence-free term of the interpolant approximates the divergence-free term of the target function, etc.

5.1. Interpolation and approximation via vector spherical polynomials. Being able to find approximation rates for target functions that are not in the native space is known as "escaping" the native space. The initial escape was first proven in the case of scalar RBFs by Narcowich, Ward, and Wendland, and their technique has since been applied to various situations involving RBFs [12, 13, 27, 28, 31]. A common theme in all these cases is to use functions that are band-limited, that is, functions whose Fourier transforms are compactly supported. For vector functions on the sphere, band-limited functions are simply vector spherical polynomials. In our case the idea is to find a band-limited function that simultaneously interpolates and approximates the target function. The goal of this section is to prove the following theorem.

TheOrem 2. Let $\tau>1$. There exists constants $\kappa$ and $C$, both of which depend only on $\tau$, such that if $L \geq \kappa / q_{X}$, then for every $\mathbf{f} \in H^{\tau}\left(\mathbb{S}^{2}\right)$ there is a vector polynomial $\mathbf{p} \in \boldsymbol{\Sigma}_{L}$ such that $\left.\mathbf{p}\right|_{X}=\left.\mathbf{f}\right|_{X}$ and $\mathbf{p}$ approximates $\mathbf{f}$ in the sense that

$$
\|\mathbf{f}-\mathbf{p}\|_{H^{\tau}\left(\mathbb{S}^{2}\right)} \leq C \operatorname{dist}_{H^{\tau}\left(\mathbb{S}^{2}\right)}\left(\mathbf{f}, \boldsymbol{\Sigma}_{L}\right) .
$$


A divergence-free variation of this theorem has been proven in [13]. However, because we are dealing with a slightly more general case, we will provide a thorough outline of the proof for the convenience of the reader. Ultimately this result relies on the following proposition, whose proof can be found in [29].

Proposition 2. Let $\mathcal{Y}$ be a (possibly complex) Banach Space, let $\mathcal{V}$ be a subspace of $\mathcal{Y}$, and let $Z^{*}$ be a finite dimensional subspace of $\mathcal{Y}^{*}$, the dual of $\mathcal{Y}$. If for every $z^{*} \in Z^{*}$ and some $\beta>1, \beta$ independent of $z^{*}$

$$
\left\|z^{*}\right\|_{\mathcal{Y}^{*}} \leq \beta\left\|z^{*} \mid \mathcal{V}\right\|_{\mathcal{V}^{*}}
$$

then for $y \in \mathcal{Y}$ there exists $v \in \mathcal{V}$ such that $v$ interpolates $y$ on $Z^{*}$; that is, $z^{*}(y)=$ $z^{*}(v)$ for all $z^{*} \in Z^{*}$. In addition, $v$ approximates $y$ in the sense that $\|y-v\|_{\mathcal{Y}} \leq$ $(1+2 \beta) \operatorname{dist}(y, \mathcal{V})$.

We will apply Proposition 2 to the following situation:

$$
\mathcal{Y}=\mathcal{N}_{\Psi}, \quad \mathcal{V}=\Sigma_{L}, \quad Z^{*}=\operatorname{span}\left\{\mathbf{c}_{j}^{T} \delta_{x_{j}}: x_{j} \in X, \mathbf{c}_{j} \in T_{x_{j}} \mathbb{S}^{2}\right\},
$$

where $\Psi$ is chosen so that $H^{\tau}\left(\mathbb{S}^{2}\right)=\mathcal{N}_{\Psi}$ with equivalent norms. Following the discussion in section 2.5, the obvious choice is to build $\Psi$ out of any RBF satisfying (10). We choose the RBF $\phi$ given by $\widehat{\phi}(\xi)=\left(1+\|\xi\|^{2}\right)^{-(\tau+3 / 2)}$, i.e., a Matérn kernel [25].

The next step is to rewrite all functionals involved in terms of their Reisz representers, and since we are dealing with native spaces, the Reisz representers can be expressed in terms of reproducing kernels. Recall that the kernel of $\mathcal{N}_{\Psi}$ is $\Psi(x, y)$, and by expanding $\Psi$ in a Fourier series, we have

$$
\Psi(x, y)=\sum_{\ell=1}^{\infty} \sum_{m=-\ell}^{\ell} \ell(\ell+1) \widehat{\psi}(\ell)\left(\mathbf{y}_{\ell, m}(x) \mathbf{y}_{\ell, m}^{T}(y)+\mathbf{z}_{\ell, m}(x) \mathbf{z}_{\ell, m}^{T}(y)\right) .
$$

From this we get a simple representation for the kernel of $H^{\tau}\left(\mathbb{S}^{2}\right) \mid \boldsymbol{\Sigma}_{L}$, given by

$$
\Psi_{L}(x, y)=\sum_{\ell=1}^{L} \sum_{m=-\ell}^{\ell} \ell(\ell+1) \widehat{\psi}(\ell)\left(\mathbf{y}_{\ell, m}(x) \mathbf{y}_{\ell, m}^{T}(y)+\mathbf{z}_{\ell, m}(x) \mathbf{z}_{\ell, m}^{T}(y)\right) .
$$

If $z^{*}=\sum \mathbf{c}_{j}^{T} \delta_{x_{j}}$, then the Riesz representers are given by $\mathbf{g}(x)=\sum_{j=1}^{N} \Psi\left(x, x_{j}\right) \mathbf{c}_{j}$ and $\mathbf{g}_{L}(x)=\sum_{j=1}^{N} \Psi_{L}\left(x, x_{j}\right) \mathbf{c}_{j}$. Now, using this and the fact that $\mathbf{g}$ and $\mathbf{g}-\mathbf{g}_{L}$ are orthogonal in $\mathcal{N}_{\Psi}$, one can show that (15) is equivalent to the estimate

$$
\frac{\left\|\mathbf{g}-\mathbf{g}_{L}\right\|_{\mathcal{N}_{\Psi}}^{2}}{\|\mathbf{g}\|_{\mathcal{N}_{\Psi}}^{2}} \leq 1-\frac{1}{\beta^{2}}
$$

Instead of estimating the above quantity directly, it is possible to "lift" the problem to $\mathbb{R}^{3}$ and use previously proven results. More specifically, from (7) and (8), we have

$$
\Psi(x, y)=P_{x}^{T} \Phi_{\text {curl }}(x, y) P_{y}+Q_{x}^{T} \Phi_{\text {curl }}(x, y) Q_{y} .
$$

As mentioned before, the kernel $\Phi_{\text {curl }}$, which gives curl-free interpolants in $\mathbb{R}^{3}$, has been studied: it is positive definite on $\mathbb{R}^{3}$, and estimates similar to (16) have been given [12]. We will use (17) to transfer the problem from $\mathcal{N}_{\Psi}$ to $\mathcal{N}_{\Phi_{\text {curl }}}$. This is encapsulated in the following lemma. 
Lemma 1. Let $X=\left\{x_{j}\right\}_{j=1}^{N}$ be a finite set of distinct points in $\mathbb{S}^{2}$, and let $\mathbf{c}_{j} \subset \mathbb{R}^{3}$ be such that $\mathbf{c}_{j} \in T_{x_{j}} \mathbb{S}^{2}$ for all $j$. Also, let $\grave{\mathbf{c}}_{j}=\mathbf{n}_{x_{j}} \times \mathbf{c}_{j}$. Then we have

$$
\left\|\sum_{j} \Psi\left(x, x_{j}\right) \mathbf{c}_{j}\right\|_{\mathcal{N}_{\Psi}}^{2}=\left\|\sum_{j} \Phi_{\text {curl }}\left(x, x_{j}\right) \mathbf{c}_{j}\right\|_{\mathcal{N}_{\Phi_{\text {curl }}}}^{2}+\left\|\sum_{j} \Phi_{\text {curl }}\left(x, x_{j}\right) \grave{\mathbf{c}}_{j}\right\|_{\mathcal{N}_{\Phi_{\text {curl }}}}^{2} .
$$

Proof. Recall that $Q_{x} \mathbf{p}=\mathbf{n}_{x} \times \mathbf{p}$ and $P_{x} \mathbf{p}=\mathbf{p}$ if $\mathbf{p} \in T_{x}\left(\mathbb{S}^{2}\right)$. Using this and standard properties of reproducing kernels, we have

$$
\begin{aligned}
\left\|\sum_{j} \Psi\left(x, x_{j}\right) \mathbf{c}_{j}\right\|_{\mathcal{N}_{\Psi}}^{2} & =\sum_{j, k} \mathbf{c}_{k}^{T} \Psi\left(x_{k}, x_{j}\right) \mathbf{c}_{j}=\sum_{j, k}\left(\mathbf{c}_{k}^{T} \Phi_{\text {curl }}\left(x_{k}, x_{j}\right) \mathbf{c}_{j}+\grave{\mathbf{c}}_{k}{ }^{T} \Phi_{\text {curl }}\left(x_{k}, x_{j}\right) \grave{\mathbf{c}}_{j}\right) \\
& =\left\|\sum_{j} \Phi_{\text {curl }}\left(x, x_{j}\right) \mathbf{c}_{j}\right\|_{\mathcal{N}_{\text {curl }}}^{2}+\left\|\sum_{j} \Phi_{\text {curl }}\left(x, x_{j}\right) \grave{\mathbf{c}}_{j}\right\|_{\mathcal{N}_{\Phi_{\text {curl }}}}^{2}
\end{aligned}
$$

This completes the proof.

The next step is to deal with lifting $\mathbf{g}_{L}$ to $\mathcal{N}_{\Phi_{\text {curl }}}$. This function is band-limited, and the results we would like to employ involve functions that are also band-limited, except in the $\mathbb{R}^{3}$ sense. However, the "lifted" version of $\mathbf{g}_{L}$ may fail to be band-limited on $\mathbb{R}^{3}$. Nevertheless, there is a way to work around this issue. Before we show this, it is necessary to introduce some notation.

Let $\sigma>0$. We let $\phi_{\sigma}$ be defined by $\widehat{\phi_{\sigma}}=\chi_{\sigma} \widehat{\phi}$, where $\chi_{\sigma}$ is the characteristic function on the ball of radius $\sigma$. Define $\Phi_{\text {curl }, \sigma}$ to be the curl-free kernel generated by $\phi_{\sigma}$, i.e., $\Phi_{c u r l, \sigma}=-\nabla \nabla^{T} \phi_{\sigma}$. We denote the SBF obtained by restricting $\phi_{\sigma}(x, y)$ to $\mathbb{S}^{2} \times \mathbb{S}^{2}$ by $\psi_{\sigma}$. Also, we let $\Psi_{d i v, \sigma}$ and $\Psi_{c u r l, \sigma}$ denote the divergence-free and curl-free SBFs generated by $\psi_{\sigma}$, respectively. Finally, to condense some of our equations we introduce the notation

$$
\begin{aligned}
\mathbf{g}_{1}(x) & :=\sum_{j} \Phi_{c u r l}\left(x, x_{j}\right) \mathbf{c}_{j}, \quad \mathbf{g}_{2}(x):=\sum_{j} \Phi_{c u r l}\left(x, x_{j}\right) \grave{\mathbf{c}}_{j}, \\
\mathbf{g}_{1, \sigma}(x) & :=\sum_{j}\left(\Phi_{\text {curl }}\left(x, x_{j}\right)-\Phi_{c u r l, \sigma}\left(x, x_{j}\right)\right) \mathbf{c}_{j} \\
\mathbf{g}_{2, \sigma}(x) & :=\sum_{j}\left(\Phi_{\text {curl }}\left(x, x_{j}\right)-\Phi_{c u r l, \sigma}\left(x, x_{j}\right)\right) \grave{\mathbf{c}}_{j} .
\end{aligned}
$$

With this notation we present the following lemma, which will allow us to circumvent the issue mentioned in the previous paragraph.

Lemma 2. There exists a constant and $L_{0}>0$ such that if $L \geq L_{0}$ and $\sigma \leq e^{-1} L$, then

$$
\left\|\mathbf{g}-\mathbf{g}_{L}\right\|_{\mathcal{N}_{\Psi}}^{2} \leq 2\left(\left\|\mathbf{g}_{1, \sigma}\right\|_{\mathcal{N}_{\Phi_{\text {curl }}}}^{2}+\left\|\mathbf{g}_{2, \sigma}\right\|_{\mathcal{N}_{\Phi_{\text {curl }}}}^{2}\right) .
$$

Proof. Using properties of reproducing kernels and the Fourier expansions of $\Psi$ and $\Psi_{L}$, it is straightforward to show the following:

$$
\left\|\mathbf{g}-\mathbf{g}_{L}\right\|_{\mathcal{N}_{\Psi}}^{2}=\sum_{\ell=L+1}^{\infty} \sum_{m=-\ell}^{\ell} \ell(\ell+1) \widehat{\psi}(\ell)\left(\left|\sum_{j=1}^{N} \mathbf{c}_{j}^{T} \mathbf{y}_{\ell, m}\left(x_{j}\right)\right|^{2}+\left|\sum_{j=1}^{N} \mathbf{c}_{j}^{T} \mathbf{z}_{\ell, m}\left(x_{j}\right)\right|^{2}\right)
$$

Copyright (c) by SIAM. Unauthorized reproduction of this article is prohibited. 
By [28, equation 4.13], there exists $L_{0}$ such that if $L \geq L_{0}$ and $\sigma \leq e^{-1} L$, we have $\widehat{\psi}(\ell) \leq 2\left(\widehat{\psi}(\ell)-\widehat{\psi}_{\sigma}(\ell)\right)$. Using this fact along with standard properties of reproducing kernels gives us

$$
\begin{aligned}
\left\|\mathbf{g}-\mathbf{g}_{L}\right\|_{\mathcal{N}_{\Psi}}^{2} & \leq 2 \sum_{\ell=L+1}^{\infty} \sum_{m=-\ell}^{\ell} \ell(\ell+1)\left(\widehat{\psi}-\widehat{\psi}_{\sigma}\right)(\ell)\left(\left|\sum_{j=1}^{N} \mathbf{c}_{j}^{T} \mathbf{y}_{\ell, m}\left(x_{j}\right)\right|^{2}+\left|\sum_{j=1}^{N} \mathbf{c}_{j}^{T} \mathbf{z}_{\ell, m}\left(x_{j}\right)\right|^{2}\right) \\
& <2 \sum_{\ell=1}^{\infty} \sum_{m=-\ell}^{\ell} \ell(\ell+1)\left(\widehat{\psi}-\widehat{\psi}_{\sigma}\right)(\ell)\left(\left|\sum_{j=1}^{N} \mathbf{c}_{j}^{T} \mathbf{y}_{\ell, m}\left(x_{j}\right)\right|^{2}+\left|\sum_{j=1}^{N} \mathbf{c}_{j}^{T} \mathbf{z}_{\ell, m}\left(x_{j}\right)\right|^{2}\right) \\
& =2\left(\sum_{j, k} \mathbf{c}_{k}^{T}\left(\Psi_{\text {div }}-\Psi_{\text {div }, \sigma}\right)\left(x_{k}, x_{j}\right) \mathbf{c}_{j}+\mathbf{c}_{k}^{T}\left(\Psi_{\text {curl }}-\Psi_{\text {curl }, \sigma}\right)\left(x_{k}, x_{j}\right) \mathbf{c}_{j}\right) \\
& =2\left(\sum_{j, k}{\grave{\mathbf{c}_{k}}}^{T}\left(\Phi_{\text {curl }}-\Phi_{\text {curl }, \sigma}\right)\left(x_{k}, x_{j}\right) \grave{\mathbf{c}}_{j}+\mathbf{c}_{k}^{T}\left(\Phi_{\text {curl }}-\Phi_{\text {curl }, \sigma}\right)\left(x_{k}, x_{j}\right) \mathbf{c}_{j}\right) \\
& =2\left(\left\|\mathbf{g}_{1, \sigma}\right\|_{\mathcal{N}_{\Phi_{\text {curl }}}}^{2}+\left\|\mathbf{g}_{2, \sigma}\right\|_{\mathcal{N}_{\Phi_{\text {curl }}}}^{2}\right) . \quad \mathbf{u}
\end{aligned}
$$

Now we have enough machinery to prove Theorem 2.

Proof of Theorem 2. Recall that the proof will follow if we can show that (16) holds for some constant $\beta$. We will be able to choose $\beta=2$. A quick application of Lemma 1 yields the identity

$$
\|\mathbf{g}\|_{\mathcal{N}_{\Psi}}^{2}=\left\|\mathbf{g}_{1}\right\|_{\mathcal{N}_{\Phi_{\text {curl }}}}^{2}+\left\|\mathbf{g}_{2}\right\|_{\mathcal{N}_{\Phi_{\text {curl }}}}^{2} .
$$

By applying Lemma 2, we get

$$
\frac{\left\|\mathbf{g}-\mathbf{g}_{L}\right\|_{\mathcal{N}_{\Psi}}^{2}}{\|\mathbf{g}\|_{\mathcal{N}_{\Psi}}^{2}} \leq 2 \frac{\left\|\mathbf{g}_{1, \sigma}\right\|_{\mathcal{N}_{\Phi_{\text {curl }}}}^{2}+\left\|\mathbf{g}_{2, \sigma}\right\|_{\mathcal{N}_{\Phi_{\text {curl }}}}^{2}}{\left\|\mathbf{g}_{1}\right\|_{\mathcal{N}_{\Phi_{\text {curl }}}}^{2}+\left\|\mathbf{g}_{2}\right\|_{\mathcal{N}_{\Phi_{\text {curl }}}}^{2}} \leq 2\left(\frac{\left\|\mathbf{g}_{1, \sigma}\right\|_{\mathcal{N}_{\Phi_{\text {curl }}}}^{2}}{\left\|\mathbf{g}_{1}\right\|_{\mathcal{N}_{\Phi_{\text {curl }}}}^{2}}+\frac{\left\|\mathbf{g}_{2, \sigma}\right\|_{\mathcal{N}_{\Phi_{\text {curl }}}}^{2}}{\left\|\mathbf{g}_{2}\right\|_{\mathcal{N}_{\Phi_{\text {curl }}}}^{2}}\right) .
$$

The ratio $\left\|\mathbf{g}_{1, \sigma}\right\|_{\mathcal{N}_{\Phi_{\text {curl }}}}^{2} /\left\|\mathbf{g}_{1}\right\|_{\mathcal{N}_{\Phi_{\text {curl }}}}^{2}$ can be measured by the same techniques used in [12, Proof of Lemma 2]. The result is

$$
\frac{\left\|\mathbf{g}_{1, \sigma}\right\|_{\mathcal{N}_{\Phi_{\text {curl }}}}^{2}}{\left\|\mathbf{g}_{1}\right\|_{\mathcal{N}_{\Phi_{\text {curl }}}}^{2}} \leq C\left(\sigma q_{X, \mathbb{R}^{3}}\right)^{2-2 \tau}
$$

where $q_{X, \mathbb{R}^{3}}$ is the separation radius of $X$ in the $\mathbb{R}^{3}$ sense, and $C$ is a constant independent of $\sigma$ and $X$. One can bound $\left\|\mathbf{g}_{2, \sigma}\right\|_{\mathcal{N}_{\Phi_{\text {curl }}}}^{2} /\left\|\mathbf{g}_{2}\right\|_{\mathcal{N}_{\Phi_{\text {curl }}}}^{2}$ similarly. The result is

$$
\frac{\left\|\mathbf{g}-\mathbf{g}_{L}\right\|_{\mathcal{N}_{\Psi}}^{2}}{\|\mathbf{g}\|_{\mathcal{N}_{\Psi}}^{2}} \leq C\left(\sigma q_{X, \mathbb{R}^{3}}\right)^{2-2 \tau}
$$

Now note that $q_{X, \mathbb{R}^{3}}$ is equivalent to $q_{X}$ up to a constant factor, and by Lemma 2 we may choose $\sigma=e^{-1} L$ to get

$$
\frac{\left\|\mathbf{g}-\mathbf{g}_{L}\right\|_{\mathcal{N}_{\Psi}}^{2}}{\|\mathbf{g}\|_{\mathcal{N}_{\Psi}}^{2}} \leq C\left(L q_{X}\right)^{2-2 \tau}
$$

Now we let $L=\kappa / q_{X}$, where $\kappa$ is chosen so large that the right-hand side is less than $3 / 4$. This is equivalent to (15) with $\beta=2$. Applying Proposition 2 and using the fact that the native space of $\Psi$ is equivalent in norm to $H^{\tau}\left(\mathbb{S}^{2}\right)$ gives us the result.

Copyright (c) by SIAM. Unauthorized reproduction of this article is prohibited. 
5.2. Interpolation error for smooth and rough target functions. Now we shift our attention to the error associated with the interpolation problem. Many of the arguments that follow have been applied in several other situations. Sobolev error estimates have been derived in a similar way for scalar- and matrix-valued RBFs and scalar SBFs [12, 27, 28, 31]. In fact, the methods we employ here have been recently used to derive similar results for the divergence-free tangent kernel $\Psi_{\text {div }}[13$, section 4].

For the first two results, we assume that all data is generated by a target function smooth enough to be within the native space of the kernel. Historically, in this case one would use the "power function" method to derive pointwise error estimates $[19,24,42]$. However, if the native space is Sobolev, one can instead use recent Sobolev estimates for functions with scattered zeros on $\mathbb{R}^{n}$. Specifically, one can use the following proposition, whose proof can be found in [30].

Proposition 3. Let $k$ be a positive integer, $0 \leq s<1$, and define $\tau=k+s$. Also, let $1 \leq p<\infty, 1 \leq q \leq \infty$, and let $\mu$ be an integer satisfying $k>\mu+n / p$, or $p=1$ and $k \geq \mu+n$. Finally, let $\Omega \subset \mathbb{R}^{n}$ be a compact set, and let $X \subset \Omega$ be a discrete set with mesh norm $h_{X, \Omega}$. Then there is a constant $C_{0}$ such that if $h_{X, \Omega} \leq C_{0}$ and if $u \in W_{p}^{\tau}\left(\mathbb{R}^{n}\right)$ satisfies $\left.u\right|_{X}=0$, then

$$
|u|_{W_{q}^{\mu}\left(\mathbb{R}^{n}\right)} \leq C h_{X, \Omega}^{\tau-\mu-n(1 / p-1 / q)_{+}}|u|_{W_{p}^{\tau}\left(\mathbb{R}^{n}\right)},
$$

where $(x)_{+}=x$ is $x \geq 0$ and is 0 otherwise. Here the constant $C$ is independent of $h_{X, \Omega}$ and $u$.

This estimate can be easily adapted from $\mathbb{R}^{n}$ to vector fields on the sphere via coordinate charts. This is the main ingredient in the following theorem. Hereafter we let $\tau, \mu$, and $q$ be as in the previous proposition. Also, so that $\mathcal{N}_{\Psi}=H^{\tau}\left(\mathbb{S}^{2}\right)$, we suppose that $\Psi$ is generated by an RBF $\phi$ satisfying (10).

THEOREM 3. Using the kernel $\Psi$, let $I_{X} \mathbf{f}$ be the interpolant to the target function $\mathbf{f}$ on the node set $X \subset \mathbb{S}^{2}$. Then we have the following error estimate:

$$
\left\|\mathbf{f}-I_{X} \mathbf{f}\right\|_{W_{q}^{\mu}\left(\mathbb{S}^{2}\right)} \leq C h_{X}^{\tau-\mu-2(1 / 2-1 / q)_{+}}\|\mathbf{f}\|_{H^{\tau}\left(\mathbb{S}^{2}\right)} .
$$

Outline of proof. Following the remarks made above, one obtains the powers of $h_{X}$ by using the fact that the error function is Sobolev with many zeros. The rest of the proof follows by using properties of the norm of the interpolation error in $\mathcal{N}_{\Psi}=H^{\tau}\left(\mathbb{S}^{2}\right)$. The proof is identical to that of [13, Theorem 4.5], with obvious modifications, so we refer the reader to that paper for further details.

If our target function is very smooth, i.e., $\mathbf{f} \in H^{\beta}\left(\mathbb{S}^{2}\right)$ with $\beta>\tau$, one gets even faster convergence. Specifically, using the standard "doubling trick" from splines gives us the following. The proof follows steps similar to the one given in [13, Corollary 4.6], so we omit the proof.

Corollary 3. Let $\mathbf{f} \in H^{2 \tau-\mu}$. Using the kernel $\Psi$, let $I_{X} \mathbf{f}$ be the interpolant to the target function $\mathbf{f}$ on the node set $X \subset \mathbb{S}^{2}$. Then we have the following error estimate:

$$
\left\|\mathbf{f}-I_{X} \mathbf{f}\right\|_{H^{\mu}\left(\mathbb{S}^{2}\right)} \leq C h_{X}^{2(\tau-\mu)}\|\mathbf{f}\|_{H^{2 \tau-\mu}\left(\mathbb{S}^{2}\right)} .
$$

We now state interpolation error estimates when the target function is less smooth than functions in the native space. The proof of this result relies on the band-limited interpolation results from section 5.1. 
TheOREm 4. Let $\tau \geq \beta>1$, and let $\phi$ be an RBF satisfying (10). Also, let $X=\left\{x_{j}\right\}_{j=1}^{N} \subset \mathbb{S}^{2}$ be a set of distinct points with mesh norm $h_{X}$, separation radius $q_{X}$, and mesh ratio $\rho_{X}=h_{X} / q_{X}$. If $\mathbf{f} \in H^{\beta}\left(\mathbb{S}^{2}\right)$, then for $0 \leq \mu \leq\lfloor\beta\rfloor-1$ we have

$$
\left\|\mathbf{f}-I_{X} \mathbf{f}\right\|_{W_{q}^{\mu}\left(\mathbb{S}^{2}\right)} \leq C \rho_{X}^{\tau-\beta} h_{X}^{\beta-\mu-2(1 / 2-1 / q)_{+}}\|\mathbf{f}\|_{H^{\beta}\left(\mathbb{S}^{2}\right)} .
$$

Outline of proof. One gets the powers of $h_{X}$ by using the fact that the error is Sobolev with many zeros:

$$
\left\|\mathbf{f}-I_{X} \mathbf{f}\right\|_{W_{q}^{\mu}\left(\mathbb{S}^{2}\right)} \leq C h_{X}^{\beta-\mu-2(1 / 2-1 / q)_{+}}\left\|\mathbf{f}-I_{X} \mathbf{f}\right\|_{H^{\beta}\left(\mathbb{S}^{2}\right)} .
$$

The remainder of the proof is to bound $\left\|\mathbf{f}-I_{X} \mathbf{f}\right\|_{H^{\beta}\left(\mathbb{S}^{2}\right)}$. The next step is to add and subtract the band-limited function $\mathbf{p}$ that interpolates $\mathbf{f}$ from Theorem 2 to the error function and apply a triangle inequality, giving

$$
\left\|\mathbf{f}-I_{X} \mathbf{f}\right\|_{H^{\beta}\left(\mathbb{S}^{2}\right)} \leq\|\mathbf{f}-\mathbf{p}\|_{H^{\beta}\left(\mathbb{S}^{2}\right)}+\left\|\mathbf{p}-I_{X} \mathbf{p}\right\|_{H^{\beta}\left(\mathbb{S}^{2}\right)} .
$$

Finally, one can apply Theorem 3, the approximation properties of $\mathbf{p}$, and a Bernstein inequality to finish the proof. For details, see the proof of [13, Theorem 4.7].

5.3. Approximation of the vector decomposition. We now come to the main result of the paper, which is to show that all approximation takes place simultaneously on each term of the Helmholtz-Hodge decomposition. We denote by $\mathcal{P}_{\text {div }}$ the $L_{2}$-projection of vector fields onto the subspace of divergence-free functions, which is defined by

$$
\left.\left.\widetilde{\left(\mathcal{P}_{d i v} \mathbf{f}\right.}\right)_{d i v}(l, m)=\widetilde{\mathbf{f}}_{d i v}(l, m) \text { and } \quad \widetilde{\left(\mathcal{P}_{d i v} \mathbf{f}\right.}\right)_{c u r l}(l, m)=0 .
$$

From this we can see that even though this projection is defined in the $L_{2}$-topology, the same linear operator is also a projection from $H^{\beta}\left(\mathbb{S}^{2}\right)$ to $H_{d i v}^{\beta}\left(\mathbb{S}^{2}\right)$ for all $\beta \geq 0$. This gives us the following inequality:

$$
\left\|\mathbf{f}_{d i v}-\left(I_{X} \mathbf{f}\right)_{d i v}\right\|_{H^{\beta}\left(\mathbb{S}^{2}\right)}=\left\|\mathcal{P}_{d i v}\left(\mathbf{f}-I_{X} \mathbf{f}\right)\right\|_{H^{\beta}\left(\mathbb{S}^{2}\right)} \leq\left\|\mathbf{f}-I_{X} \mathbf{f}\right\|_{H^{\beta}\left(\mathbb{S}^{2}\right)} .
$$

A similar estimate holds for curl-free functions. With this simple observation the result follows as a corollary to Theorem 4 and Corollary 3.

Theorem 5. Let $\tau \geq \beta>1$, and let $\phi$ be an RBF satisfying (10). Also, let $X=\left\{x_{j}\right\}_{j=1}^{N} \subset \mathbb{S}^{2}$ be a set of distinct points with mesh norm $h_{X}$, separation radius $q_{X}$, and mesh ratio $\rho_{X}=h_{X} / q_{X}$. If $\mathbf{f} \in H^{\beta}\left(\mathbb{S}^{2}\right)$, then for $0 \leq \mu \leq\lfloor\beta\rfloor-1$ we have the following error estimates:

$$
\begin{aligned}
\left\|\mathbf{f}_{\text {div }}-\left(I_{X} \mathbf{f}\right)_{d i v}\right\|_{H^{\mu}\left(\mathbb{S}^{2}\right)} & \leq C \rho_{X}^{\tau-\beta} h_{X}^{\beta-\mu}\|\mathbf{f}\|_{H^{\beta}\left(\mathbb{S}^{2}\right)}, \\
\left\|\mathbf{f}_{\text {curl }}-\left(I_{X} \mathbf{f}\right)_{\text {curl }}\right\|_{H^{\mu}\left(\mathbb{S}^{2}\right)} & \leq C \rho_{X}^{\tau-\beta} h_{X}^{\beta-\mu}\|\mathbf{f}\|_{H^{\beta}\left(\mathbb{S}^{2}\right)} .
\end{aligned}
$$

Furthermore, if $\mathbf{f} \in H^{2 \tau-\mu}\left(\mathbb{S}^{2}\right)$, we have the following error estimates:

$$
\begin{aligned}
&\left\|\mathbf{f}_{d i v}-\left(I_{X} \mathbf{f}\right)_{d i v}\right\|_{H^{\mu}\left(\mathbb{S}^{2}\right)} \leq C h_{X}^{2(\tau-\mu)}\|\mathbf{f}\|_{H^{2 \tau-\mu}\left(\mathbb{S}^{2}\right)}, \\
&\left\|\mathbf{f}_{\text {curl }}-\left(I_{X} \mathbf{f}\right)_{\text {curl }}\right\|_{H^{\mu}\left(\mathbb{S}^{2}\right)} \leq C h_{X}^{2(\tau-\mu)}\|\mathbf{f}\|_{H^{2 \tau-\mu}\left(\mathbb{S}^{2}\right)} .
\end{aligned}
$$

6. Numerical results. Numerical results presented in this section verify the above predicted results for the stability (Corollary 2) and the Sobolev error estimates (Corollary 3 and Theorems 4 and 5) for the vector SBF interpolant and its decomposition. The setup for the numerical experiments is similar to the setup in [13] for verifying the estimates for the divergence-free SBF interpolants.

Copyright $@$ by SIAM. Unauthorized reproduction of this article is prohibited. 
6.1. Kernels. To test the stability and error estimates, we use four different kernels $\Psi$, which are generated from the restriction to the sphere of two different classes of positive definite RBFs: Matérn and Wendland.

The Matérn (or Sobolev spline) functions were introduced for applications in [25], and are arguably the most popular and most important family of kernels for statistical applications [15]. They are defined by

$$
\mathrm{MA}_{\nu}: \phi(r)=\frac{2^{1-\nu}}{\Gamma(\nu)}(\varepsilon r)^{\nu} K_{\nu}(\varepsilon r)
$$

where $K_{\nu}$ corresponds to the $K$-Bessel function of order $\nu$ and $\varepsilon>0$ is the free shape parameter. In $\mathbb{R}^{3}$, the Fourier transform of $\phi$ decays like

$$
\widehat{\phi}(\xi) \sim\left(1+\|\xi\|_{2}^{2}\right)^{-\left(\nu+\frac{3}{2}\right)} .
$$

Thus, the tangent kernel $\Psi$ generated from $\mathrm{MA}_{\nu}$ gives rise to the native space $\mathcal{N}_{\Psi}=$ $H^{\nu}(\mathbb{S})$.

The Wendland functions were first introduced in [37] and have also been used successfully in many applications [38]. They are tailored to be positive definite, compactly supported, and of a specific smoothness in the particular dimension $n$ that the underlying approximation problem is posed in. Their general form is given by

$$
\mathrm{WE}_{n, k}: \phi(r)= \begin{cases}p_{n, k}(r), & 0 \leq r \leq \delta, \\ 0, & r>\delta,\end{cases}
$$

where $p_{n, k}$ is a polynomial of degree $\lfloor n / 2\rfloor+3 k+1, \phi \in C^{2 k}$, and $\delta>0$ is the support radius. In $\mathbb{R}^{3}$, the Fourier transform of $\phi$ decays like [38, p. 157]

$$
\widehat{\phi}(\xi) \sim\left(1+\|\xi\|_{2}^{2}\right)^{-\left(\left(k+\frac{1}{2}\right)+\frac{3}{2}\right)},
$$

which makes the tangent kernel $\Psi$ generated from $p_{n, k}$ satisfy $\mathcal{N}_{\Psi}=H^{k+\frac{1}{2}}(\mathbb{S})$.

The exact forms of the four RBFs used to generate the tangent kernels $\Psi$ in the numerical experiments are listed in Table 1. Also included in this table is the corresponding native space of $\Psi$ for each of the RBFs.

TABLE 1

\begin{tabular}{|c|c|c|}
\hline & RBFs for generating $\Psi$ in the numerical experiments & $\mathcal{N}_{\Psi}$ \\
\hline 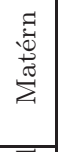 & $\begin{array}{l}\mathrm{MA}_{7 / 2}: \quad \phi(r)=e^{-\varepsilon r}\left(1+(\varepsilon r)+\frac{2}{5}(\varepsilon r)^{2}+\frac{1}{15}(\varepsilon r)^{3}\right), \\
\mathrm{MA}_{9 / 2}: \quad \phi(r)=e^{-\varepsilon r}\left(1+(\varepsilon r)+\frac{3}{7}(\varepsilon r)^{2}+\frac{2}{21}(\varepsilon r)^{3}+(\varepsilon r)^{4}\right)\end{array}$ & $\begin{array}{l}H^{\frac{7}{2}}(\mathbb{S}) \\
H^{\frac{9}{2}}(\mathbb{S})\end{array}$ \\
\hline 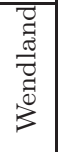 & $\begin{array}{ll}\mathrm{WE}_{3,3}: & \phi(r)=\left(1-\frac{r}{\delta}\right)_{+}^{8}\left(1+8 \frac{r}{\delta}+25\left(\frac{r}{\delta}\right)^{2}+32\left(\frac{r}{\delta}\right)^{3}\right), \\
\mathrm{WE}_{3,4}: & \phi(r)=\left(1-\frac{r}{\delta}\right)_{+}^{10}\left(1+10 \frac{r}{\delta}+42\left(\frac{r}{\delta}\right)^{2}+90\left(\frac{r}{\delta}\right)^{3}+\frac{429}{5}\left(\frac{r}{\delta}\right)^{4}\right)\end{array}$ & $\begin{array}{l}H^{\frac{7}{2}}(\mathbb{S}) \\
H^{\frac{9}{2}}(\mathbb{S})\end{array}$ \\
\hline
\end{tabular}

The RBFs used for generating the tangent kernels $\Psi=\Psi_{\text {div }}+\Psi_{\text {curl }}$ for the numerical examples. For Matérn, $\varepsilon>0$ is called the shape parameter while $\delta>0$ is called the support radius for Wendland.

As is the case with many RBFs, the Matérn and Wendland classes both feature a free parameter ( $\varepsilon$ and $\delta$, respectively). The choice for these parameters can have 
a considerable effect on the stability and accuracy of the RBF interpolant. However, determining the "optimal" value for either of these parameters is neither easy nor obvious (cf. $[4,7,33]$ in the case of the shape parameter, and [38, Ch. 15], [18, Ch. 5] in the case of the support radius). In the numerical results that follow, we make no attempt to optimize these values and fix them at $\varepsilon=4$ for $\mathrm{MA}_{7 / 2}, \varepsilon=8$ for $\mathrm{MA}_{9 / 2}$, $\delta=5 / 3$ for $\mathrm{WE}_{3,3}$, and $\delta=4 / 3$ for $\mathrm{WE}_{3,4}$. We leave the investigation of optimal parameter selection for vector SBF interpolation to a separate study.

6.2. Node sets. As in the two previous works $[32,13]$, we use the minimum energy (ME) node sets of Womersley and Sloan [41] for our numerical examples since they have several nice properties. First, both the mesh-norm $h_{X}$ and the separation radius $q_{X}$ for these node sets decay approximately uniformly like the inverse of the square root of the number of nodes $N$, i.e., $h_{X}, q_{X} \sim \frac{1}{\sqrt{N}}$; see Figure 2. This means that the mesh ratio $\rho_{X}=h_{X} / q_{X}$ appearing in the error estimate from Theorems 4 and 5 stays roughly constant as $N$ is increased. Second, the nodes are not oriented along any vertices or lines, which emphasizes the ability of the vector SBF technique to handle arbitrary node layouts. Third, many of these node sets are freely available for download on the web [41].

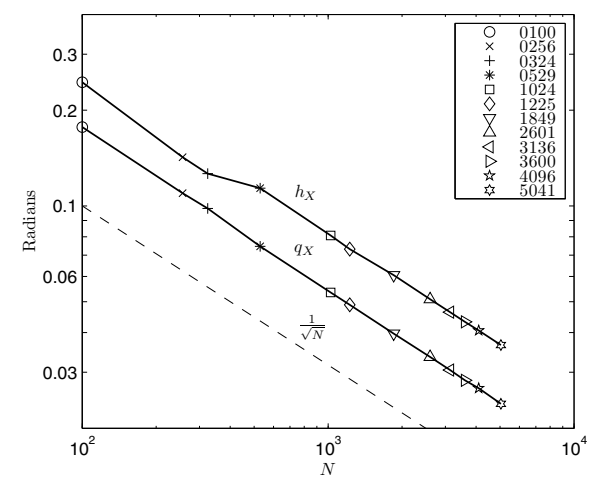

FIG. 2. Mesh-norm $h_{X}$ and separation radius $q_{X}$ for minimum energy (ME) nodes of varying sizes $N$ as indicated by the legend. The dashed line shows the line $1 / \sqrt{N}$.

6.3. Verification of stability estimates. For the $\mathrm{MA}_{7 / 2}$ and $\mathrm{WE}_{3,3}$ kernels, Corollary 2 predicts that the minimum eigenvalue of the vector SBF interpolation matrices $A_{X, \Psi}$ decreases like $q_{X}^{5}$ as $q_{X}$ is decreased. The corollary similarly predicts that the decrease in the minimum eigenvalue is $q_{X}^{7}$ for the $\mathrm{MA}_{9 / 2}$ and $\mathrm{WE}_{4,3}$ kernels. To test these estimates, we construct the vector SBF interpolation matrices for each of the kernels and for several ME node sets and compute their corresponding minimum eigenvalue. Figure 3 displays the results on a log-log scale as a function of the separation radius of the ME node sets. Also included in the figure are the predicted rates of decrease (as dashed and dash-dotted lines). It is clear from the figure that Corollary 2 provides a very accurate estimate on the behavior of the minimum eigenvalue of $A_{X, \Psi}$.

We also numerically verified the estimates from Corollary 1 on the minimum eigenvalue of just the curl-free SBF interpolation matrix. However, we have not included them here since they are very similar to the div-free results from [13]. 


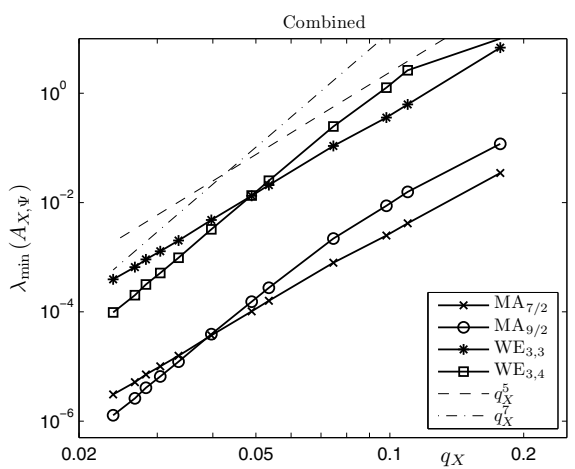

FIG. 3. Minimum eigenvalue of the vector $S B F$ interpolation matrices $A_{X, \Psi}$ as a function of the separation radius $q_{X}$ of the $M E$ node sets (note the log-log scale). The dashed line is the predicted estimate from Corollary 2 for the kernels based on $M A_{\frac{7}{2}}$ and $W E_{3,3}$, while the dash-dotted line is the prediction for the kernels based on $M A_{\frac{9}{2}}$ and $W E_{3,4}$.

6.4. Verification of error estimates. To verify the error estimates from Corollary 3 and Theorems 4 and 5, we use three vector fields of varying smoothness. All the fields are generated using "stream functions" and "velocity potentials" so that we can easily separate the divergence-free and curl-free parts of the field. Letting the scalar functions $s$ and $v$ denote the respective stream function and velocity potential, each of the vector fields will be given by

$$
\mathbf{f}=\underbrace{\mathbf{L} s}_{\mathbf{f}_{d i v}}+\underbrace{\nabla_{*} v}_{\mathbf{f}_{c u r l}} \text {. }
$$

Recall from section 2 that $\mathbf{L}$ and $\nabla_{*}$ denote the surface curl and surface gradient, respectively. Thus, $\mathbf{L} s$ is divergence-free, while $\nabla_{*} v$ is curl-free.

In the descriptions of the stream functions and velocity potentials below, we use a mix of intrinsic (spherical) and extrinsic (Cartesian) coordinates. For the former, we let $\lambda$ denote the longitudinal direction $(-\pi \leq \lambda<\pi)$ and $\theta$ denote the latitudinal direction, which we measure from the equator (i.e., $-\pi / 2 \leq \theta \leq \pi / 2$ ). For the latter, we let $x$ be a point on the sphere with Cartesian components $\left(x_{1}, x_{2}, x_{3}\right)$. We also remind the reader that the operators $\mathbf{L}$ and $\nabla_{*}$ can easily be expressed as $Q_{x} \nabla$ and $P_{x} \nabla$, respectively, where $\nabla$ is the gradient operator in $\mathbb{R}^{3}$ and $Q_{x}$ and $P_{x}$ are defined in section 2.4 .

To measure the error, vector SBF interpolants are constructed for each of the three vector fields based on samples of these fields at several different ME node sets. These interpolants and target functions are then evaluated and compared at a much denser set of points. For these evaluation points we use the 16,641 minimum determinant (MD) node set of Womersley and Sloan [41], which provides a quasi-uniform discretization of the sphere. Finally, the (relative) discrete $\ell_{2}$-norm of the error, which is based on the inner product (2), is computed using the surface-quadrature weights that are included with the 16,641 MD node set. This will give a similar measure of the error as the continuous Sobolev norms in Corollary 3 and Theorems 4 and 5 for the case of $\mu=0$ [30].

Field 1. The stream function and velocity potential for the first field are linear combinations of spherical harmonics and are meant to generate realistic synoptic scale 
meteorological wind fields. The definition of spherical harmonics we adhere to is $Y_{\ell, m}(x)=\sqrt{\frac{(2 l+1)}{4 \pi} \frac{(\ell-|m|) !}{(\ell+|m|) !}} P_{\ell,|m|}\left(x_{3}\right) \begin{cases}\cos \left(|m| \tan ^{-1}\left(\frac{x_{2}}{x_{1}}\right)\right), & m=0, \ldots, \ell, \\ \sin \left(|m| \tan ^{-1}\left(\frac{x_{2}}{x_{1}}\right)\right), & m=-\ell, \ldots,-1,\end{cases}$ where $P_{\ell, m}$ are the associated Legendre functions.

The stream function is given by

$$
s_{1}(x)=-\frac{1}{\sqrt{3}} Y_{1,0}(x)+\frac{8 \sqrt{2}}{3 \sqrt{385}} Y_{5,4}(x),
$$

which is known as a Rosby-Haurwitz wave and is an analytic solution to the nonlinear barotropic vorticity equation on the sphere [17, pp. 453-454]. It is also used as the initial condition for one of the de facto test cases for the shallow water wave equations on the sphere [40].

For the velocity potential, we use

$$
v_{1}(x)=\frac{1}{25}\left[Y_{4,0}(x)+Y_{6,-3}(x)\right] .
$$

The order of the spherical harmonics and coefficients here is chosen somewhat arbitrarily; what is desired is a curl-free field with an interesting pattern, but of less strength since for synoptic scale motions of the atmosphere, the wind is nearly nondivergent [17, pp. 386]. The fields generated by each of these scalar potentials, as well as the combined field f , are plotted in Figure 4(a).

Since $\mathbf{f}$ is $C^{\infty}\left(\mathbb{S}^{2}\right)$, we expect the "doubling" estimate from Corollary 3 to apply to the vector SBF interpolants. Similarly, we expect the second result from Theorem 5 to apply to the error estimate on the vector SBF recovery of the decomposition. For the $\mathrm{MA}_{7 / 2}$ and $\mathrm{WE}_{3,3}$ kernels, the error is expected to decrease like $h_{X}^{7}$ for the combined and decomposed fields, while it is expected to decrease like $h_{X}^{9}$ in case of the $\mathrm{MA}_{9 / 2}$ and $\mathrm{WE}_{3,4}$ kernels. In Figure 5(a), the relative $\ell_{2}$ errors for the combined and decomposed fields are displayed in a sequence of three plots. The predicted results for each field are also displayed (see the dashed and dashed-dotted lines) and a very good agreement with the actual errors is clear.

Field 2. For the second field we again use the Rosby-Haurwitz wave (20) as the stream function, while we use a linear combination of compactly supported functions for the velocity potential. For the compactly supported functions we use cubic $B$ splines, which can be defined as follows. Let $x$ and $x_{c}$ be points on the unit sphere, where $x_{c}$ has spherical coordinates $\left(\theta_{c}, \lambda_{c}\right)$, and define the (Euclidean) distance from $x$ to $x_{c}$ as $r=\sqrt{\left.2-2 x \cdot x_{c}\right)}$. Then

$$
f\left(x ; \sigma, \theta_{c}, \lambda_{c}\right)=\frac{\sigma^{3}}{12} \sum_{j=0}^{4}(-1)^{j}\left(\begin{array}{l}
4 \\
j
\end{array}\right)\left|r-\frac{(j-2)}{\sigma}\right|^{3}
$$

is a cubic $B$-spline in $r$ with center $x_{c}$ (or $\left(\theta_{c}, \lambda_{c}\right)$ ) and is positive for $|r|<2 / \sigma$ and zero elsewhere. We use the following combinations of $f$ for the velocity potential:

$$
\begin{aligned}
v_{2}(x)=\frac{1}{8} f(x ; 10 / 2, \pi / 6,0) & -\frac{1}{7} f(x ; 3, \pi / 5,-\pi / 7)+\frac{1}{9} f(x ; 10 / 2,-\pi / 6, \pi, 2) \\
& -\frac{1}{8} f(x ; 3,-\pi / 5, \pi / 3) .
\end{aligned}
$$

Copyright $@$ by SIAM. Unauthorized reproduction of this article is prohibited. 


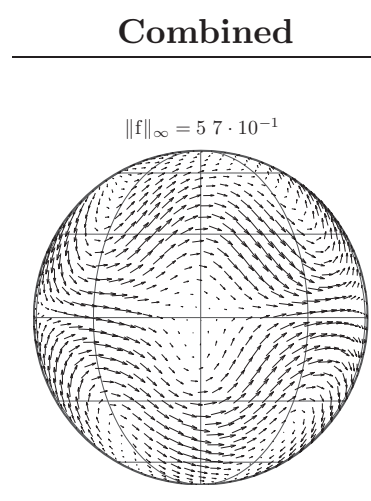

\section{$=\quad$ Div.-Free}

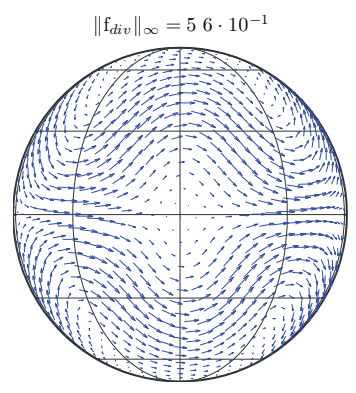

(a) Field 1
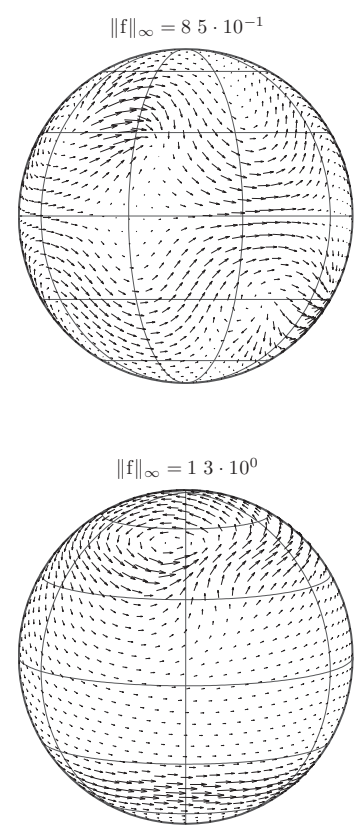

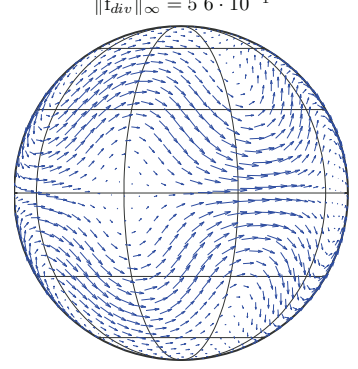

(b) Field 2

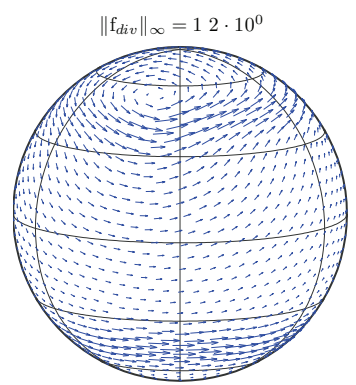

(c) Field 3 $\left\|\mathrm{f}_{\text {div }}\right\|_{\infty}=56 \cdot 10^{-1}$
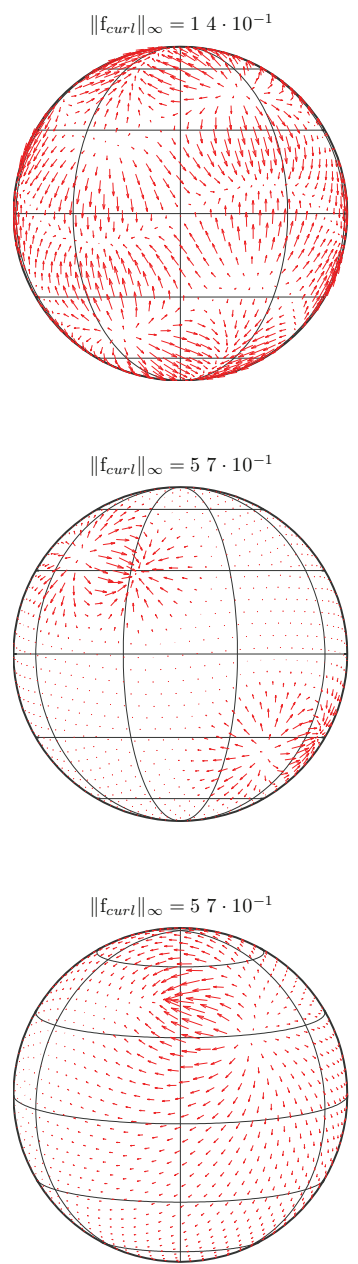

FIG. 4. (a)-(c) The vector fields used in the numerical examples. The first column is the field that is sampled for the interpolation procedure. All plots are orthographic projections of the fields sampled at $N=1849 \mathrm{ME}$ nodes and displayed from the following $(\theta, \lambda)$ viewpoint: (a) (0,0), (b) $(0, \pi / 9),(\mathrm{c})(\pi / 18,0)$. For display purposes, each field has been normalized by its max norm.

As explained in [13], $f$ is in the Sobolev space $H^{\tau}\left(\mathbb{S}^{2}\right)$ for all $\tau<4$. Thus, the curlfree field generated by $v_{2}$ is in $H_{\text {curl }}^{\beta}\left(\mathbb{S}^{2}\right)$ and the combined field $\mathbf{f}$ is in $H^{\beta}\left(\mathbb{S}^{2}\right)$ for all $\beta<3$. Figure 4(b) displays the combined Field 2, as well as the fields generated by each of the scalar potentials.

For this field, the estimates from Theorems 4 and 5 apply to the vector SBF interpolant and its decomposition, respectively. These theorems predict that the errors for $\mathrm{MA}_{7 / 2}$ and $\mathrm{WE}_{3,3}$ should decrease like $\rho_{X}^{1 / 2} h_{X}^{3}$ and like $\rho_{X}^{3 / 2} h_{X}^{3}$ for the $\mathrm{MA}_{7 / 2}$ and $\mathrm{WE}_{3,3}$ kernels. However, since the mesh ratio $\rho_{X}$ is kept nearly constant 


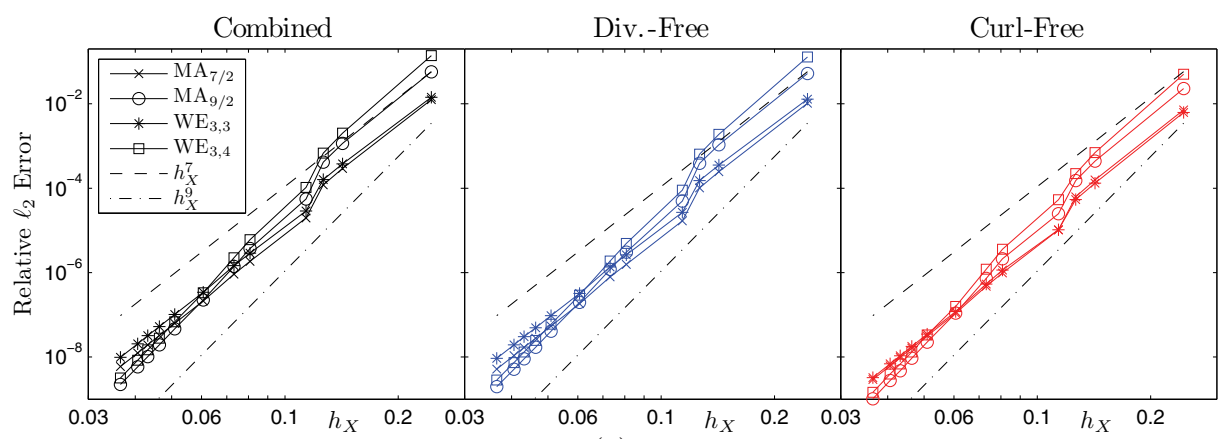

(a)

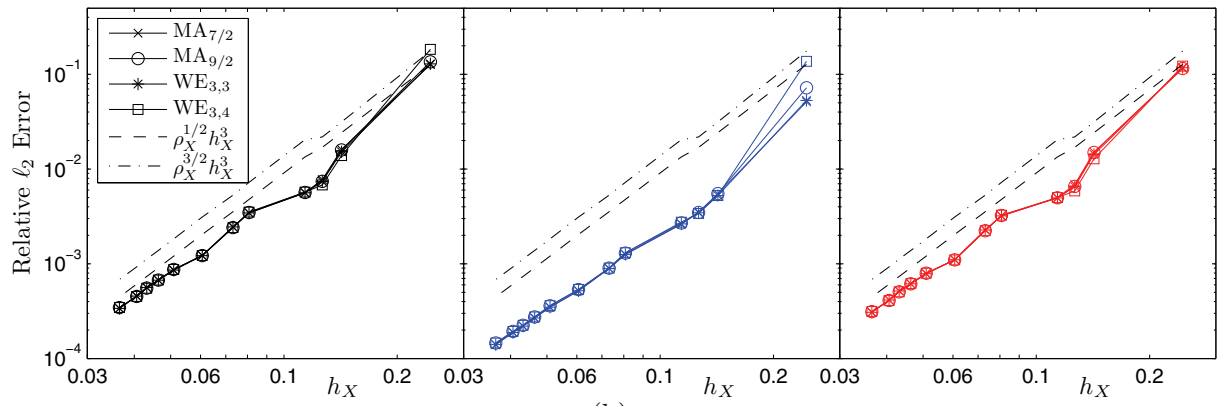

(b)

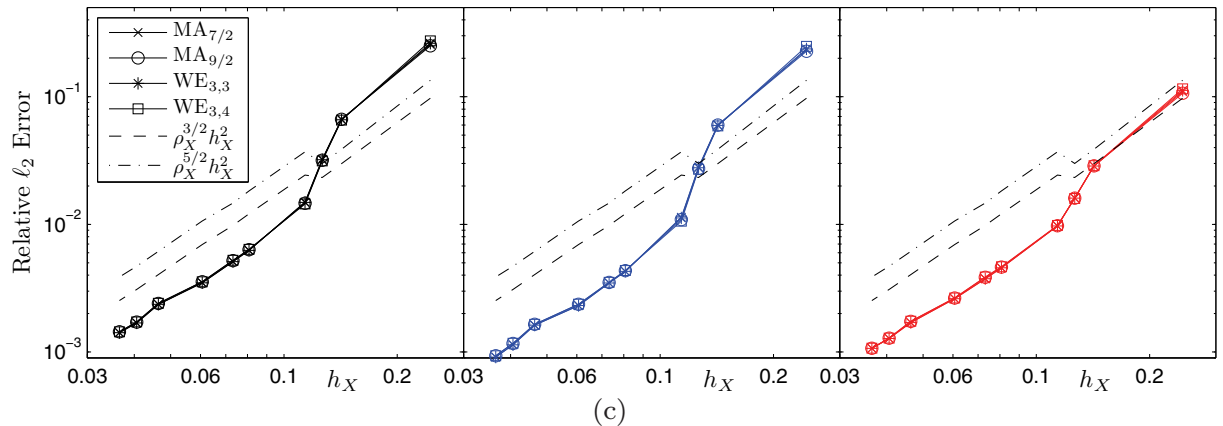

FIG. 5. (a)-(c) Relative $\ell_{2}$-errors as a function of the mesh-norm $h_{X}$ of the $M E$ node sets for the vector decomposed RBF interpolants to the vector fields shown in Figure 4(a)-(c). The dashed and dash-dotted lines in each figure are defined by the plot legend and are included for comparison purposes with the theoretical results. The vertical limits on each row are the same, and both the horizontal and vertical scales are logarithmic.

as the resolution is increased, the dependence on $\rho_{X}$ will not be evident. The actual relative $\ell_{2}$ errors for all four of the kernels are displayed in Figure 5(b). It is again clear that the predicted results are closely aligned with the true results. It is also interesting to note that although the divergence-free field is infinitely smooth, we see that the error does not decrease faster than what the smoothness of the sampled field allows.

Field 3. Let $x_{c} \in \mathbb{S}^{2}$ have spherical coordinates $\left(\theta_{c}, \lambda_{c}\right)$, and let $t=x \cdot x_{c}$ and 
$a=1-t$. Define

$$
\begin{aligned}
g\left(x ; \theta_{c}, \lambda_{c}\right) & =-\frac{1}{2}\left[\left(3 t+3 \sqrt{2} a^{3 / 2}-4\right)+\left(3 t^{2}-4 t+1\right) \log (a)\right. \\
& +(3 t-1) a \log (\sqrt{2 a}+a)] .
\end{aligned}
$$

This function is referred to as the "spherical spline" of order 2 and is in the Sobolev space $H^{\tau}\left(\mathbb{S}^{2}\right)$ for all $\tau<3[13]$.

The stream function for Field 3 is defined using $g$ as follows:

$$
s_{3}(x)=\int_{-\pi / 2}^{\theta} \sin ^{14}(2 \xi) d \xi-3 g(x ; \pi / 4,-\pi / 12),
$$

where $\theta$ is the latitudinal coordinate of $x$. Since $g$ controls the smoothness of $s_{3}$, the divergence-free field generated by $s_{3}$ is in $H_{d i v}^{\beta}\left(\mathbb{S}^{2}\right)$ for all $\beta<2$. This field models a low pressure system in a jet stream that is symmetrical about the equator and is similar to the wind field used in Test Case 4 of [40], although our field is much less smooth.

The velocity potential is also defined using $g$ as follows:

$$
v_{3}(x)=\frac{5}{2} g(x ; \pi / 4,0)-\frac{7}{4} g(x ; \pi / 6, \pi, 9)-\frac{3}{2} g(x ; 5 \pi / 16, \pi / 10) .
$$

The curl-free field generated from $v$ is similarly in $H_{\text {curl }}^{\beta}\left(\mathbb{S}^{2}\right)$ for $\beta<3$. The combined field generated by $v$ and $s$ will thus be in $H^{\beta}\left(\mathbb{S}^{2}\right)$ for $\beta<3$. This field and its two components are displayed in Figure 4(c).

Theorems 4 and 5 also apply to the respective vector $\mathrm{SBF}$ interpolant and its decomposition. The predicted decrease in the errors for the $\mathrm{MA}_{7 / 2}$ and $\mathrm{WE}_{3,3}$ kernels is $\rho_{X}^{3 / 2} h_{X}^{2}$ and is $\rho_{X}^{5 / 2} h_{X}^{2}$ for $\mathrm{MA}_{7 / 2}$ and $\mathrm{WE}_{3,3}$. Like Field 2, however, the dependence on $\rho_{X}$ is not evident. Figure 5(c) displays the actual relative $\ell_{2}$ errors for all four of the kernels. As with the previous two fields, it is again clear that the true results match the predicted ones very well.

7. Concluding remarks. We have introduced a new numerical technique for fitting and decomposing a tangent vector field on $\mathbb{S}^{2}$ using RBFs. The method can be used for both scattered and gridded data and suffers from no pole singularities. In the case of interpolation, we have presented results on the stability and approximation properties of the technique. For stability, we have obtained optimal estimates on the spectral condition number of the interpolation matrices involved in the method and verified these estimates numerically. For the approximation properties, we have obtained Sobolev estimates on the interpolant and its decomposition for vector fields in the underlying native space of the tangent kernels, smoother than the native space, and in the very important case of fields too rough for the native space. We have again presented numerical results verifying these estimates using vector fields with characteristics similar to those of synoptic scale horizontal winds.

We conclude with some remarks on the computational cost of the method. To determine the interpolation coefficients for $N$ samples of a vector field, the $2 N \times$ $2 N$ linear system (14) must be solved. For globally supported kernels, this system will be dense and thus direct methods for inverting it (as used in this study) will be practical only for a moderate size $N$. For the compactly supported kernels, the linear system (14) can be made sparse and thus computationally cheaper to invert. 
However, as is well-known in the RBF literature, one must be careful in balancing the goodness of fit with the support of these basis functions [38, p. 185]. Iterative methods for inverting the linear systems, such as Krylov subspace methods [34], will be practical only for large data sets if the matrix vector products can be computed efficiently (i.e., in $O(N)$ or $O(N \log N)$ operations) and if a good preconditioner is used. These computational issues are also prevalent in standard RBF interpolation methods and much work has gone into successfully overcoming them (cf. [38, Ch. 15] and the references therein). An obvious first step in addressing the computational deficiencies of the present method for large data sets is to use these already established techniques.

\section{REFERENCES}

[1] S. Beth, Multiscale Approximation by Vector Radial Basis Functions on the Sphere, Ph.D. thesis, University of Kaiserslautern, Kaiserslautern, Germany, 2000.

[2] S. C. Brenner and L. Ridgway Scott, The Mathematical Theory of Finite Element Methods, 2nd ed., Texts Appl. Math. 15, Springer-Verlag, New York, 2002.

[3] R. Daley, Atmospheric Data Analysis, Cambridge University Press, Cambridge, 1991.

[4] G. E. Fasshauer and J. G. Zhang, On choosing "optimal" shape parameters for RBF approximation, Numer. Algorithms, 45 (2007), pp. 345-368.

[5] M. J. Fengler and W. Freeden, A nonlinear Galerkin scheme involving vector and tensor spherical harmonics for solving the incompressible Navier-Stokes equation on the sphere, SIAM J. Sci. Comput., 27 (2005), pp. 967-994.

[6] N. Flyer And G. B. Wright, A radial basis function method for the shallow water equations on a sphere, Proc. R. Soc. Lond. Ser. A, 465 (2009), pp. 1949-1976.

[7] B. Fornberg And J. Zuev, The Runge phenomenon and spatially variable shape parameters in RBF interpolation, Comput. Math. Appl., 54 (2007), pp. 379-398.

[8] W. Freeden And T. Gervens, Vector spherical spline interpolation-basic theory and computational aspects, Math. Methods Appl. Sci., 16 (1993), pp. 151-183.

[9] W. Freeden, T. Gervens, and M. Schreiner, Constructive Approximation on the Sphere, in Numer. Math. Sci. Comput., The Clarendon Press, Oxford University Press, New York, 1998.

[10] W. Freeden and M. Schreiner, Spherical Functions of Mathematical Geosciences: A Scalar, Vectorial, and Tensorial Setup, Advances in Geophysical and Environmental Mechanics and Mathematics, Springer, New York, 2009.

[11] E. J. Fuselier, Improved stability estimates and a characterization of the native space for matrix-valued RBFs, Adv. Comput. Math., 29 (2008), pp. 269-290.

[12] E. J. FuselieR, Sobolev-type approximation rates for divergence-free and curl-free RBF interpolants, Math. Comp., 77 (2008), pp. 1407-1423.

[13] E. J. Fuselier, F. J. Narcowich, J. D. Ward, and G. B. Wright, Error and stability estimates for surface-divergence free RBF interpolants on the sphere, Math. Comp., 78 (2009), pp. 2157-2186.

[14] P. B. Gilkey, The Index Theorem and the Heat Equation, Notes by Jon Sacks, Mathematics Lecture Series, No. 4, Publish or Perish Inc., Boston, MA, 1974.

[15] T. Gneiting, Correlation functions for atmospheric data analysis, Q. J. R. Meteorol. Soc., 125 (1999), pp. 2449-2464.

[16] G. J. Haltiner and R. T. Williams, Numerical Prediction and Dynamic Meteorology, 2nd ed., John Wiley and Sons, New York, 1980.

[17] J. R. Holton, An Introduction to Dynamic Meteorology, 3rd ed., Academic Press, San Francisco, 1992.

[18] A. Iske, Multiresolution Methods in Scattered Data Modelling, Lect. Notes Comput. Sci. Eng. 37, Springer-Verlag, Berlin, 2004.

[19] K. Jetter, J. StÖckler, And J. D. WARD, Error estimates for scattered data interpolation on spheres, Math. Comp., 68 (1999), pp. 733-747.

[20] T. N. Krishnamurti And L. Bounoua, An Introduction to Numerical Weather Prediction Techniques, CRC Press, Boca Raton, FL, 1996.

[21] J.-L. Lions And E. Magenes, Nonhomogeneous Boundary Value Problems and Applications, translated from the French by P. Kenneth, Die Grundlehren der Mathematischen Wissenschaften, Band 181, Springer-Verlag, New York, 1972. 
[22] P. Lynch, Deducing the wind from vorticity and divergence, Mon. Wea. Rev., 116 (1988), pp. 86-93.

[23] C. L. Mader, Numerical Modeling of Water Waves, CRC Press, Boca Raton, FL, 2004.

[24] W. R. MAdych And S. A. Nelson, Multivariate interpolation and conditionally positive definite functions, Approx. Theory Appl., 4 (1988), pp. 77-89.

[25] B. Matérn, Spatial Variation, 2nd ed., with a Swedish summary, Lecture Notes Statist. 36, Springer-Verlag, Berlin, 1986.

[26] C. MülLeR, Spherical Harmonics, Lecture Notes in Math. 17, Springer-Verlag, Berlin-New York, 1966.

[27] F. J. NARCOWICh, X. Sun, AND J. D. WARD, Approximation power of RBFs and their associated SBFs: A connection, Adv. Comput. Math., 27 (2007), pp. 107-124.

[28] F. J. Narcowich, X. Sun, J. D. Ward, and H. Wendland, Direct and inverse Sobolev error estimates for scattered data interpolation via spherical basis functions, Found. Comput. Math., 7 (2007), pp. 369-390.

[29] F. J. NARcowich AND J. D. WARD, Scattered-data interpolation on $\mathbb{R}^{n}$ : Error estimates for radial basis and band-limited functions, SIAM J. Math. Anal., 36 (2004), pp. 284-300.

[30] F. J. Narcowich, J. D. Ward, And H. Wendland, Sobolev bounds on functions with scattered zeros, with applications to radial basis function surface fitting, Math. Comp., 74 (2005), pp. $743-763$.

[31] F. J. NARCOWich, J. D. Ward, And H. Wendland, Sobolev error estimates and a Bernstein inequality for scattered data interpolation via radial basis functions, Constr. Approx., 24 (2006), pp. $175-186$.

[32] F. J. Narcowich, J. D. Ward, and G. B. Wright, Divergence-free RBFs on surfaces, J. Fourier Anal. Appl., 13 (2007), pp. 643-663.

[33] S. RIPPA, An algorithm for selecting a good value for the parameter $c$ in radial basis function interpolation, Adv. Comput. Math., 11 (1999), pp. 193-210.

[34] Y. SAAD, Iterative Methods for Sparse Linear Systems, 2nd ed., SIAM, Philadelphia, 2003.

[35] G. WAHBA, Vector splines on the sphere, with application to the estimation of vorticity and divergence from discrete, noisy data, in Multivariate Approximation Theory, II (Oberwolfach, 1982), Internat. Ser. Numer. Math. 61, Birkhäuser, Basel, 1982, pp. 407-429.

[36] I. G. Watterson, Decomposition of global ocean currents using a simple iterative method, J. Atmos. Oceanic Technol., 18 (2001), pp. 691-703.

[37] H. Wendland, Piecewise polynomial, positive definite and compactly supported radial functions of minimal degree, Adv. Comput. Math., 4 (1995), pp. 389-396.

[38] H. Wendland, Scattered Data Approximation, Cambridge Monogr. Appl. Comput. Math. 17, Cambridge University Press, Cambridge, UK, 2005.

[39] D. L. Williamson, The evolution of dynamical cores for global atmospheric models, J. Meteorol. Soc. Jpn., 85B (2007), pp. 241-269.

[40] D. L. Williamson, J. B. Drake, J. J. Hack, R. Jakob, and P. N. Swarztrauber, A standard test set for numerical approximations to the shallow water equations in spherical geometry, J. Comput. Phys., 102 (1992), pp. 211-224.

[41] R. S. Womersley and I. H. Sloan, Interpolation and Cubature on the Sphere, available online at http://web.maths.unsw.edu.au/ rsw/Sphere/.

[42] Z. M. WU AND R. SchabaCK, Local error estimates for radial basis function interpolation of scattered data, IMA J. Numer. Anal., 13 (1993), pp. 13-27.

Copyright (c) by SIAM. Unauthorized reproduction of this article is prohibited. 